JarosŁaW L. Bojarski (Warszawa)

\title{
GENERAL METHOD OF REGULARIZATION. II: RELAXATION PROPOSED BY SUQUET
}

Abstract. The aim of this paper is to prove that the relaxation of the elastic-perfectly plastic energy (of a solid made of a Hencky material) is the lower semicontinuous regularization of the plastic energy. We find the integral representation of a non-locally coercive functional. We show that the set of solutions of the relaxed problem is equal to the set of solutions of the relaxed problem proposed by Suquet. Moreover, we prove an existence theorem for the limit analysis problem.

1. Introduction. In the first part of this paper (see [2]) we investigate the convex functional

$$
B D \ni \mathbf{u} \mapsto \mathbb{B}(\varepsilon(\mathbf{u}))=\int_{\bar{\Omega}} h(x, \varepsilon(\mathbf{u}))
$$

with constraints on the boundary of $\Omega$, where $\varepsilon(\mathbf{u})$ is the symmetrized gradient of $\mathbf{u}$ and $B D(\Omega)$ is the space of bounded deformations. Moreover, we assume that $\mathbb{B}(\varepsilon(\mathbf{u}))=\infty$ if $\varepsilon(\mathbf{u}) \notin L^{1}$. In [1] we find the lower semicontinuous (l.s.c.) relaxation of $\mathbb{B}$, and we show that the relaxation is a l.s.c. function (in the weak* $B D$ topology), not greater than $\mathbb{B}$. Here we prove that the above mentioned relaxation (in the case of the relaxation proposed by Suquet) is the largest 1.s.c. minorant less than $\mathbb{B}$, i.e. it is the l.s.c. regularization of $\mathbb{B}$. If the volume forces are 0 , then we can omit the assumption of global coercivity of the functional considered. Moreover, we show that the set of solutions of the classical relaxed problem is equal to the set of solutions of the relaxed problem proposed by Suquet.

2000 Mathematics Subject Classification: Primary 49J45; Secondary 49K99, 47N10, 47H04, 26B30, 74C05.

Key words and phrases: regularization (relaxation), functions of bounded deformation, integral representation, Hencky plasticity, limit analysis problem.

Supported by Committee for Scientific Research (Poland) grant No. 2P03A00518. 
In [3], the global method for relaxation is applied for l.s.c. regularization of quasiconvex functionals with constraints (Dirichlet condition). These functionals are defined on $B V(\Omega)$. The constraints considered do not describe the relaxation proposed by Suquet (see [12]).

In [6] and [7] Christiansen finds the solution for the limit analysis problem, associated to the relaxed problem proposed by Suquet. But the limit analysis problem is not explicitly formulated in [6] and the relation between solutions of the relaxed problem and solutions of the relaxed problem proposed by Suquet is not considered.

The classical method of relaxation does not allow one to find a solution of the limit analysis problem $\left(P_{0}\right)_{A L}$ (see [13], [1]). Therefore we study the method of relaxation proposed by Suquet (cf. [12], [1]).

In Section 3, we obtain an existence theorem for the limit analysis problem, associated to the relaxed problem proposed by Suquet. In Corollary 10, we get a criterion of coercivity of the original problem $\left(P_{\lambda, j}\right)$, or the relaxed problem $\left(R P_{\lambda, j}^{* *}\right)$ (see $[2,(3.9)-(3.11),(5.1),(5.3),(5.4)$ and $\left.(5.8)]\right)$.

In the Appendix, we describe the scheme of duality in convex optimization in the case of Hencky plasticity.

We obtain the above mentioned results under the following assumptions.

Assumption $1 . \Omega$ and $\Omega_{1}$ are bounded open connected sets of class $C^{1}$ in $\mathbb{R}^{n}$ such that $\Omega \subset \subset \Omega_{1}$.

Let $\Gamma_{0}$ and $\Gamma_{1}\left(=\bar{\Gamma}_{1}\right)$ be Borel subsets of the boundary $\operatorname{Fr} \Omega$ of $\Omega$ such that $\Gamma_{0} \cap \Gamma_{1}=\emptyset$ and $d s\left(\operatorname{Fr} \Omega-\left(\Gamma_{0} \cup \Gamma_{1}\right)\right)=0$. The Lebesgue and the Hausdorff measures on $\Omega$ and $\operatorname{Fr} \Omega$ are denoted by $d x$ and $d s$, respectively.

In this paper we consider the Banach space of measurable functions

$$
W^{n}(\Omega, \operatorname{div}) \equiv\left\{\boldsymbol{\sigma} \in L^{\infty}\left(\Omega, \mathbf{E}_{s}^{n}\right) \mid \operatorname{div} \boldsymbol{\sigma} \in L^{n}\left(\Omega, \mathbb{R}^{n}\right)\right\}
$$

with the natural norm $\|\boldsymbol{\sigma}\|_{W^{n}(\Omega, \operatorname{div})}=\|\boldsymbol{\sigma}\|_{L^{\infty}\left(\Omega, \mathbf{E}_{s}^{n}\right)}+\|\operatorname{div} \boldsymbol{\sigma}\|_{L^{n}\left(\Omega, \mathbb{R}^{n}\right)}$ (where $\mathbf{E}_{s}^{n}$ is the space of symmetric real $n \times n$ matrices). Moreover, we consider the space

$$
C_{\text {div }}\left(\bar{\Omega}, \mathbf{E}_{s}^{n}\right) \equiv\left\{\boldsymbol{\sigma} \in C\left(\bar{\Omega}, \mathbf{E}_{s}^{n}\right) \mid \boldsymbol{\sigma}_{\mid \operatorname{int} \Omega} \in W^{n}(\Omega, \operatorname{div})\right\} .
$$

Let $\mathcal{K}: \bar{\Omega} \rightarrow 2^{\mathbf{E}_{s}^{n}}$ be a multifunction.

Assumption 2 (see $[11$, p. 19, Lemma 1$]$ ). $\mathcal{K}(x)$ is a convex and closed subset of $\mathbf{E}_{s}^{n}$ for all $x \in \bar{\Omega}$, and there exists $\mathbf{z}_{0} \in C^{1}\left(\bar{\Omega}, \mathbf{E}_{s}^{n}\right)$ such that

$$
\mathbf{z}_{0}(x) \in \mathcal{K}(x) \quad \text { for every } x \in \bar{\Omega},
$$

and the following conditions hold:

(i) if $\mathbf{z}(x) \in \mathcal{K}(x)$ for $d x$-a.e. $x \in \Omega$, where $\mathbf{z} \in C_{\text {div }}\left(\bar{\Omega}, \mathbf{E}_{s}^{n}\right)$, then $\mathbf{z}(y) \in \mathcal{K}(y)$ for every $y \in \bar{\Omega}$; 
(ii) for every $y \in \bar{\Omega}$ and $\mathbf{w} \in \mathcal{K}(y)$ there exists $\mathbf{z} \in C_{\operatorname{div}}\left(\bar{\Omega}, \mathbf{E}_{s}^{n}\right)$ such that $\mathbf{z}(y)=\mathbf{w}$ and $\mathbf{z}(x) \in \mathcal{K}(x)$ for every $x \in \bar{\Omega}$. $y \in \bar{\Omega}$,

Conditions (i) and (ii) are equivalent to the condition that for every

$$
\mathcal{K}(y)=\left\{\mathbf{z}(y) \mid \mathbf{z} \in C_{\mathrm{div}}\left(\bar{\Omega}, \mathbf{E}_{s}^{n}\right), \mathbf{z}(x) \in \mathcal{K}(x) \text { for } d x \text {-a.e. } x \in \Omega\right\} .
$$

Definition 1. Let $j^{*}: \Omega \times \mathbf{E}_{s}^{n} \rightarrow \mathbb{R} \cup\{\infty\}$ be a convex normal integrand, i.e.

(a) the function $\mathbf{E}_{s}^{n} \ni \mathbf{w}^{*} \mapsto j^{*}\left(x, \mathbf{w}^{*}\right)$ is convex and l.s.c. for $d x$-a.e. $x \in \Omega$;

(b) there exists a Borel function $\widetilde{j^{*}}: \Omega \times \mathbf{E}_{s}^{n} \rightarrow \mathbb{R} \cup\{\infty\}$ such that $\widetilde{j^{*}}(x, \cdot)=j^{*}(x, \cdot)$ for $d x$-a.e. $x \in \Omega$.

Moreover, assume that

$$
\left\{\mathbf{w}^{*} \in \mathbf{E}_{s}^{n} \mid j^{*}\left(x, \mathbf{w}^{*}\right)<\infty\right\}=\mathcal{K}(x) \quad \text { for } d x \text {-a.e. } x \in \Omega .
$$

Assumption 3. For every $\widehat{r}>0$ there exists $c_{\widehat{r}}$ such that

$$
\begin{aligned}
\sup \left\{\int_{\Omega} j^{*}\left(x, \mathbf{z}^{*}\right) d x \mid \mathbf{z}^{*}\right. & \in L^{\infty}\left(\Omega, \mathbf{E}_{s}^{n}\right),\left\|\mathbf{z}^{*}\right\|_{L^{\infty}}<\widehat{r} \\
\text { and } \mathbf{z}^{*}(x) & \in \mathcal{K}(x) \text { for } d x \text {-a.e. } x \in \Omega\}<c_{\widehat{r}}<\infty .
\end{aligned}
$$

Assumption 4. There exist $\mathbf{u}^{e} \in L D(\Omega)$ and $q \in L^{1}(\Omega, \mathbb{R})$ such that

$$
j^{*}\left(x, \mathbf{w}^{*}\right) \geq \varepsilon\left(\mathbf{u}^{e}\right)(x): \mathbf{w}^{*}+q(x)
$$

for $d x$-a.e. $x \in \Omega$ and every $\mathbf{w}^{*} \in \mathbf{E}_{s}^{n}$, and $\gamma_{B}\left(\mathbf{u}^{e}\right)=\mathbf{0}$ on $\operatorname{Fr} \Omega$.

The set $\mathcal{K}(x)$ denotes the elasticity convex domain at the point $x$. Define

$$
j(x, \mathbf{w}) \equiv j^{* *}(x, \mathbf{w}) \equiv \sup \left\{\mathbf{w}: \mathbf{w}^{*}-j^{*}\left(x, \mathbf{w}^{*}\right) \mid \mathbf{w}^{*} \in \mathbf{E}_{s}^{n}\right\}
$$

for $d x$-a.e. $x \in \Omega$ and all $\mathbf{w} \in \mathbf{E}_{s}^{n}$. Then $j$ is a convex normal integrand. Define $j_{\infty}: \bar{\Omega} \times \mathbf{E}_{s}^{n} \rightarrow \mathbb{R} \cup\{\infty\}$ by

$$
j_{\infty}(x, \mathbf{w}) \equiv \sup \left\{\mathbf{w}: \mathbf{w}^{*}-I_{\mathcal{K}(x)}\left(\mathbf{w}^{*}\right) \mid \mathbf{w}^{*} \in \mathbf{E}_{s}^{n}\right\}
$$

for $x \in \bar{\Omega}$ and $\mathbf{w} \in \mathbf{E}_{s}^{n}$.

Assumption 5. There exists $\boldsymbol{\sigma}_{0} \in C_{\mathrm{div}}\left(\bar{\Omega}, \mathbf{E}_{s}^{n}\right)$ such that $\boldsymbol{\beta}_{B}\left(\boldsymbol{\sigma}_{0}\right)=\lambda \mathbf{g}$ on $\Gamma_{1}$ and $\sigma_{0}(x) \in \mathcal{K}(x)$ for $d x$-a.e. $x \in \Omega$, where $\mathbf{g}$ is a boundary force on $\Gamma_{1}$.

Assumption 6. Let $\Gamma_{1}=\operatorname{Fr} \Omega \cap \mathcal{C}$, where $\mathcal{C}=\operatorname{cl} \operatorname{int} \mathcal{C} \subset \Omega_{1}$ is a closed Caccioppoli set and $d s(\operatorname{Fr} \Omega \cap \operatorname{Fr} \mathcal{C})=0$.

2. Relaxed problem proposed by Suquet. The classical method of relaxation does not allow one to find a solution of the limit analysis problem $\left(P_{0}\right)_{A L}$ (see [4], [13], [1]). The problem has an interesting mechanical 
interpretation as the safety condition. Therefore we turn to the following method of relaxation, proposed by Suquet (cf. [12], [1]).

We define the following Banach spaces:

$$
\begin{aligned}
& L D(\Omega) \equiv\left\{\mathbf{u} \in L^{1}\left(\Omega, \mathbb{R}^{n}\right) \mid\right. \\
&\left.\varepsilon_{i j}(\mathbf{u}) \equiv \frac{1}{2}\left(\frac{\partial u_{i}}{\partial x_{j}}+\frac{\partial u_{j}}{\partial x_{i}}\right) \in L^{1}(\Omega), i, j=1, \ldots, n\right\}, \\
& B D(\Omega) \equiv\left\{\mathbf{u} \in L^{1}\left(\Omega, \mathbb{R}^{n}\right) \mid \varepsilon_{i j}(\mathbf{u}) \in \mathbb{M}_{b}(\Omega, \mathbb{R}), i, j=1, \ldots, n\right\},
\end{aligned}
$$

with the natural norms

$$
\begin{aligned}
\|\mathbf{u}\|_{L D} & =\|\mathbf{u}\|_{L^{1}}+\sum_{i, j}^{n}\left\|\varepsilon_{i j}(\mathbf{u})\right\|_{L^{1}}, \\
\|\mathbf{u}\|_{B D} & =\|\mathbf{u}\|_{L^{1}}+\sum_{i, j}^{n}\left\|\varepsilon_{i j}(\mathbf{u})\right\|_{\mathbb{M}_{b}},
\end{aligned}
$$

where $\mathbb{M}_{b}(\Omega, \mathbb{R})$ is the space of $\mathbb{R}$-valued bounded measures defined on $\Omega$. Moreover, we consider the space

$$
\begin{aligned}
\mathbf{Y}^{1}(\bar{\Omega}) \equiv\left\{\mathbf{M} \in \mathbb{M}_{b}\left(\bar{\Omega}, \mathbf{E}_{s}^{n}\right) \mid \exists \mathbf{u}_{1}\right. & \in B D\left(\Omega_{1}\right), \\
& \left.\boldsymbol{\varepsilon}\left(\mathbf{u}_{1}\right)_{\mid \bar{\Omega}}=\mathbf{M}, \mathbf{u}_{1 \mid \Omega_{1}-\bar{\Omega}}=\mathbf{0}\right\} .
\end{aligned}
$$

We now define the functional of the total elastic-perfectly plastic energy $\mathbb{H}_{\lambda}^{j}$, introduced in [1]. We then find its l.s.c. regularization in the topology $\sigma\left(\mathbf{Y}^{1}(\bar{\Omega}) \times \mathbf{M}^{1}\left(\Gamma_{1}\right), C_{\text {div }}\left(\bar{\Omega}, \mathbf{E}_{s}^{n}\right) \times C\left(\Gamma_{1}, \mathbf{E}_{s}^{n}\right)\right.$ ) (where the bilinear form is defined in (2.8) below). Let

$$
\mathbf{M}^{1}\left(\Gamma_{1}\right) \equiv\left\{\boldsymbol{\mu} \otimes_{s} \boldsymbol{\nu} \in \mathbb{M}_{b}\left(\Gamma_{1}, \mathbf{E}_{s}^{n}\right) \mid \boldsymbol{\mu} \in \mathbb{M}_{b}\left(\Gamma_{1}, \mathbb{R}^{n}\right)\right\}
$$

where $\boldsymbol{\nu}$ is the outer unit vector, normal to $\operatorname{Fr} \Omega$. Consider the topological vector spaces

$$
\left[C\left(\Gamma_{1}, \mathbf{E}_{s}^{n}\right), \sigma\left(C\left(\Gamma_{1}, \mathbf{E}_{s}^{n}\right), \mathbf{M}^{1}\left(\Gamma_{1}\right)\right)\right],\left[\mathbf{M}^{1}\left(\Gamma_{1}\right), \sigma\left(\mathbf{M}^{1}\left(\Gamma_{1}\right), C\left(\Gamma_{1}, \mathbf{E}_{s}^{n}\right)\right)\right]
$$

(the latter is equivalent to $\left[\mathbf{M}^{1}\left(\Gamma_{1}\right)\right.$, weak $\left.{ }^{*} \mathbf{M}^{1}\left(\Gamma_{1}\right)\right]$ ).

We define the functional $\mathbb{H}_{\lambda}^{j}: \mathbf{Y}^{1}(\bar{\Omega}) \times \mathbf{M}^{1}\left(\Gamma_{1}\right) \rightarrow \mathbb{R} \cup\{\infty\}$ by

$$
\begin{aligned}
\mathbb{H}_{\lambda}^{j}\left(\varepsilon(\mathbf{u})_{\mid \bar{\Omega}}, \boldsymbol{\mu} \otimes_{s} \boldsymbol{\nu}\right) \equiv & -\int_{\Gamma_{1}} \lambda \mathbf{g} \cdot \boldsymbol{\mu}+\int_{\Omega} j\left(x, \boldsymbol{\varepsilon}(\mathbf{u})_{a}\right) d x \\
& +\int_{\Gamma_{0}} I_{\left\{\gamma_{B}^{I}(\mathbf{u}) \otimes_{s} \boldsymbol{\nu}=\mathbf{0}\right\}}\left(\gamma_{B}^{I}(\mathbf{u}) \otimes_{s} \boldsymbol{\nu}\right) d s
\end{aligned}
$$

if $\mathbf{u}_{\mid \Omega} \in L D(\Omega)$ and $\boldsymbol{\mu}=\boldsymbol{\gamma}_{B}^{I}(\mathbf{u}) d s$ for $\left|\boldsymbol{\mu}-\boldsymbol{\gamma}_{B}^{I}(\mathbf{u}) d s\right|$-a.e. $x \in \Gamma_{1}$, and $\mathbb{H}_{\lambda}^{j}\left(\varepsilon(\mathbf{u})_{\mid \bar{\Omega}}, \boldsymbol{\mu} \otimes_{s} \boldsymbol{\nu}\right) \equiv \infty$ otherwise. Here $I_{\left\{\gamma_{B}^{I}(\mathbf{u}) \otimes_{s} \boldsymbol{\nu}=\mathbf{0}\right\}}\left(\boldsymbol{\gamma}_{B}^{I}(\mathbf{u}) \otimes_{s} \boldsymbol{\nu}\right)$ is the indicator function, which takes value 0 if $\gamma_{B}^{I}(\mathbf{u}) \otimes_{s} \boldsymbol{\nu}=\mathbf{0}$ and $\infty$ otherwise. 
We assume that there exist $\widetilde{\mathbf{u}} \in B D\left(\Omega_{1}\right)$ and $\widetilde{\boldsymbol{\mu}} \in \mathbb{M}_{b}\left(\Gamma_{1}, \mathbb{R}^{n}\right)$ such that $\widetilde{\mathbf{u}}_{\mid \Omega} \in L D(\Omega)$ and $\mathbb{H}_{\lambda}^{j}\left(\varepsilon(\widetilde{\mathbf{u}})_{\mid \bar{\Omega}}, \widetilde{\boldsymbol{\mu}} \otimes_{s} \boldsymbol{\nu}\right)<\infty$.

The bilinear form between $\mathbf{Y}^{1}(\bar{\Omega}) \times \mathbf{M}^{1}\left(\Gamma_{1}\right)$ and $C_{\text {div }}\left(\bar{\Omega}, \mathbf{E}_{s}^{n}\right) \times C\left(\Gamma_{1}, \mathbf{E}_{s}^{n}\right)$ is given by

$$
\begin{aligned}
\left\langle\left(\varepsilon(\mathbf{u})_{\mid \bar{\Omega}}, \boldsymbol{\mu} \otimes_{s} \boldsymbol{\nu}\right) ;(\boldsymbol{\sigma}, \boldsymbol{\kappa})\right\rangle_{2} & \equiv \int_{\Omega} \boldsymbol{\sigma}: \boldsymbol{\varepsilon}(\mathbf{u})_{\mid \Omega} \\
& +\int_{\operatorname{Fr} \Omega} \boldsymbol{\sigma}:\left(-\gamma_{B}^{I}(\mathbf{u}) \otimes_{s} \boldsymbol{\nu}\right) d s+\int_{\Gamma_{1}} \boldsymbol{\kappa}:\left[\boldsymbol{\mu} \otimes_{s} \boldsymbol{\nu}\right]
\end{aligned}
$$

for $\left(\varepsilon(\mathbf{u})_{\mid \Omega},-\gamma_{B}^{I}(\mathbf{u}) d s \otimes_{s} \boldsymbol{\nu}\right) \in \mathbf{Y}^{1}(\bar{\Omega}), \boldsymbol{\mu} \otimes_{s} \boldsymbol{\nu} \in \mathbf{M}^{1}\left(\Gamma_{1}\right), \boldsymbol{\sigma} \in C_{\operatorname{div}}\left(\bar{\Omega}, \mathbf{E}_{s}^{n}\right)$ and $\boldsymbol{\kappa} \in C\left(\Gamma_{1}, \mathbf{E}_{s}^{n}\right)$. Because of the duality between $\mathbf{Y}^{1}(\bar{\Omega}) \times \mathbf{M}^{1}\left(\Gamma_{1}\right)$ and $C_{\text {div }}\left(\bar{\Omega}, \mathbf{E}_{s}^{n}\right) \times C\left(\Gamma_{1}, \mathbf{E}_{s}^{n}\right)$, we define a functional $\left(\mathbb{H}_{\lambda}^{j}\right)^{*}: C_{\operatorname{div}}\left(\bar{\Omega}, \mathbf{E}_{s}^{n}\right) \times$ $C\left(\Gamma_{1}, \mathbf{E}_{s}^{n}\right) \rightarrow \mathbb{R} \cup\{\infty\}$ by

$$
\begin{aligned}
& \left(\mathbb{H}_{\lambda}^{j}\right)^{*}(\boldsymbol{\sigma}, \boldsymbol{\kappa})=\sup \left\{\left\langle\left(\varepsilon(\mathbf{u})_{\mid \bar{\Omega}}, \boldsymbol{\mu} \otimes_{s} \boldsymbol{\nu}\right) ;(\boldsymbol{\sigma}, \boldsymbol{\kappa})\right\rangle_{2}\right. \\
& \left.\quad-\mathbb{H}_{\lambda}^{j}\left(\varepsilon(\mathbf{u})_{\mid \bar{\Omega}}, \boldsymbol{\mu} \otimes_{s} \boldsymbol{\nu}\right) \mid \boldsymbol{\varepsilon}(\mathbf{u})_{\mid \bar{\Omega}} \in \mathbf{Y}^{1}(\bar{\Omega}), \boldsymbol{\mu} \otimes_{s} \boldsymbol{\nu} \in \mathbf{M}^{1}\left(\Gamma_{1}\right)\right\} .
\end{aligned}
$$

The bidual functional $\left(\mathbb{H}_{\lambda}^{j}\right)^{* *}: \mathbf{Y}^{1}(\bar{\Omega}) \times \mathbf{M}^{1}\left(\Gamma_{1}\right) \rightarrow \mathbb{R} \cup\{\infty\}$ is defined by

$$
\begin{aligned}
\left(\mathbb{H}_{\lambda}^{j}\right)^{* *}\left(\varepsilon(\mathbf{u})_{\mid \bar{\Omega}}, \boldsymbol{\mu} \otimes_{s} \boldsymbol{\nu}\right)=\sup \left\{\left\langle\left(\varepsilon(\mathbf{u})_{\mid \bar{\Omega}}, \boldsymbol{\mu} \otimes_{s} \boldsymbol{\nu}\right) ;(\boldsymbol{\sigma}, \boldsymbol{\kappa})\right\rangle_{2}\right. \\
\left.-\left(\mathbb{H}_{\lambda}^{j}\right)^{*}(\boldsymbol{\sigma}, \boldsymbol{\kappa}) \mid \boldsymbol{\sigma} \in C_{\operatorname{div}}\left(\bar{\Omega}, \mathbf{E}_{s}^{n}\right), \boldsymbol{\kappa} \in C\left(\Gamma_{1}, \mathbf{E}_{s}^{n}\right)\right\} .
\end{aligned}
$$

The bilinear form between $\mathbb{M}_{b}\left(\Omega, \mathbf{E}_{s}^{n}\right) \times \mathbf{Y}^{1}(\bar{\Omega})_{\mid \operatorname{Fr} \Omega} \times \mathbf{M}^{1}\left(\Gamma_{1}\right)$ and $C_{\text {div }}\left(\bar{\Omega}, \mathbf{E}_{s}^{n}\right) \times C\left(\Gamma_{1}, \mathbf{E}_{s}^{n}\right)$ is given by

$$
\begin{aligned}
\left\langle\left(\mathbf{w}, \boldsymbol{\gamma}_{B}^{I}(\mathbf{u}) \otimes_{s} \boldsymbol{\nu}, \boldsymbol{\mu} \otimes_{s} \boldsymbol{\nu}\right) ;(\boldsymbol{\sigma}, \boldsymbol{\kappa})\right\rangle_{3}=\int_{\Omega} \boldsymbol{\sigma}: \mathbf{w} \\
\quad+\int_{\operatorname{Fr} \Omega} \boldsymbol{\sigma}:\left(-\gamma_{B}^{I}(\mathbf{u}) \otimes_{s} \boldsymbol{\nu}\right) d s+\int_{\Gamma_{1}} \boldsymbol{\kappa}:\left[\boldsymbol{\mu} \otimes_{s} \boldsymbol{\nu}\right]
\end{aligned}
$$

for $\mathbf{w} \in \mathbb{M}_{b}\left(\Omega, \mathbf{E}_{s}^{n}\right), \gamma_{B}^{I}(\mathbf{u}) d s \otimes_{s} \boldsymbol{\nu} \in \mathbf{Y}^{1}(\bar{\Omega})_{\mid \operatorname{Fr} \Omega}, \boldsymbol{\mu} \otimes_{s} \boldsymbol{\nu} \in \mathbf{M}^{1}\left(\Gamma_{1}\right), \boldsymbol{\sigma} \in$ $C_{\text {div }}\left(\bar{\Omega}, \mathbf{E}_{s}^{n}\right)$ and $\boldsymbol{\kappa} \in C\left(\Gamma_{1}, \mathbf{E}_{s}^{n}\right)$. The extension of $\mathbb{H}_{\lambda}^{j}$ onto the space $\mathbb{M}_{b}\left(\Omega, \mathbf{E}_{s}^{n}\right) \times \mathbf{Y}^{1}(\bar{\Omega})_{\mid \operatorname{Fr} \Omega} \times \mathbf{M}^{1}\left(\Gamma_{1}\right)$ (denoted by $\left.\widetilde{\mathbb{H}}_{\lambda}^{j}\right)$ is given by

$$
\begin{aligned}
\widetilde{\mathbb{H}}_{\lambda}^{j}\left(\mathbf{w},-\gamma_{B}^{I}(\mathbf{u}) \otimes_{s} \boldsymbol{\nu}, \boldsymbol{\mu} \otimes_{s} \boldsymbol{\nu}\right) \equiv-\int_{\Gamma_{1}} \lambda \mathbf{g} \cdot \boldsymbol{\mu} \\
+\int_{\Omega} j(x, \mathbf{w}) d x+\int_{\Gamma_{0}} I_{\left\{\gamma_{B}^{I}(\mathbf{u}) \otimes_{s} \boldsymbol{\nu}=\mathbf{0}\right\}}\left(\gamma_{B}^{I}(\mathbf{u}) \otimes_{s} \boldsymbol{\nu}\right) d s
\end{aligned}
$$

if $\mathbf{w} \in L^{1}\left(\Omega, \mathbf{E}_{s}^{n}\right)$ and $\boldsymbol{\mu}=\gamma_{B}^{I}(\mathbf{u}) d s$ for $\left|\boldsymbol{\mu}-\boldsymbol{\gamma}_{B}^{I}(\mathbf{u}) d s\right|$-a.e. $x \in \Gamma_{1}$, and $\widetilde{\mathbb{H}}_{\lambda}^{j}\left(\mathbf{w},-\gamma_{B}^{I}(\mathbf{u}) \otimes_{s} \boldsymbol{\nu}, \boldsymbol{\mu} \otimes_{s} \boldsymbol{\nu}\right) \equiv \infty$ otherwise.

By duality between $\mathbb{M}_{b}\left(\Omega, \mathbf{E}_{s}^{n}\right) \times \mathbf{Y}^{1}(\bar{\Omega})_{\mid \operatorname{Fr} \Omega} \times \mathbf{M}^{1}\left(\Gamma_{1}\right)$ and $C_{\operatorname{div}}\left(\bar{\Omega}, \mathbf{E}_{s}^{n}\right) \times$ $C\left(\Gamma_{1}, \mathbf{E}_{s}^{n}\right)$, we define a functional $\left(\widetilde{\mathbb{H}}_{\lambda}^{j}\right)^{*}: C_{\text {div }}\left(\bar{\Omega}, \mathbf{E}_{s}^{n}\right) \times C\left(\Gamma_{1}, \mathbf{E}_{s}^{n}\right) \rightarrow \mathbb{R} \cup\{\infty\}$ 
by

$$
\begin{aligned}
&\left(\widetilde{\mathbb{H}}_{\lambda}^{j}\right)^{*}(\boldsymbol{\sigma}, \boldsymbol{\kappa})=\sup \{\left\langle\left(\mathbf{w},-\gamma_{B}^{I}(\mathbf{u}) \otimes_{s} \boldsymbol{\nu}, \boldsymbol{\mu} \otimes_{s} \boldsymbol{\nu}\right) ;(\boldsymbol{\sigma}, \boldsymbol{\kappa})\right\rangle_{3} \\
&-\widetilde{\mathbb{H}}_{\lambda}^{j}\left(\mathbf{w},-\gamma_{B}^{I}(\mathbf{u}) \otimes_{s} \boldsymbol{\nu}, \boldsymbol{\mu} \otimes_{s} \boldsymbol{\nu}\right) \mid \mathbf{w} \in L^{1}\left(\Omega, \mathbf{E}_{s}^{n}\right), \\
&\left.\mathbf{u} \in B D(\Omega) \text { and } \boldsymbol{\mu} \otimes_{s} \boldsymbol{\nu} \in \mathbf{M}^{1}\left(\Gamma_{1}\right)\right\},
\end{aligned}
$$

since $L^{1} \subset \mathbb{M}_{b}$. The bidual functional $\left(\widetilde{\mathbb{H}}_{\lambda}^{j}\right)^{* *}: \mathbf{Y}^{1}(\bar{\Omega}) \times \mathbf{M}^{1}\left(\Gamma_{1}\right) \rightarrow \mathbb{R} \cup\{\infty\}$ is defined by

$$
\begin{array}{r}
\left(\widetilde{\mathbb{H}}_{\lambda}^{j}\right)^{* *}\left(\mathbf{w},-\boldsymbol{\gamma}_{B}^{I}(\mathbf{u}) \otimes_{s} \boldsymbol{\nu}, \boldsymbol{\mu} \otimes_{s} \boldsymbol{\nu}\right)=\sup \left\{\left\langle\left(\mathbf{w},-\gamma_{B}^{I}(\mathbf{u}) \otimes_{s} \boldsymbol{\nu}, \boldsymbol{\mu} \otimes_{s} \boldsymbol{\nu}\right)\right.\right. \\
\left.(\boldsymbol{\sigma}, \boldsymbol{\kappa})\rangle_{3}-\left(\widetilde{\mathbb{H}}_{\lambda}^{j}\right)^{*}(\boldsymbol{\sigma}, \boldsymbol{\kappa}) \mid \boldsymbol{\sigma} \in C_{\operatorname{div}}\left(\bar{\Omega}, \mathbf{E}_{s}^{n}\right), \boldsymbol{\kappa} \in C\left(\Gamma_{1}, \mathbf{E}_{s}^{n}\right)\right\}
\end{array}
$$

for $\left(\mathbf{w},-\gamma_{B}^{I}(\mathbf{u}) d s \otimes_{s} \boldsymbol{\nu}\right) \in \mathbf{Y}^{1}(\bar{\Omega})$ and $\boldsymbol{\mu} \otimes_{s} \boldsymbol{\nu} \in \mathbf{M}^{1}\left(\Gamma_{1}\right)$.

LEMMA 1. For every $\mathbf{u} \in B D\left(\Omega_{1}\right)$ and $\boldsymbol{\mu} \in \mathbb{M}_{b}\left(\Gamma_{1}, \mathbb{R}^{n}\right)$ such that $\mathbf{u}_{\mid \Omega} \in$ $L D(\Omega), \mathbf{u}_{\mid \Omega_{1}-\bar{\Omega}}=\mathbf{0}, \gamma_{B}^{I}(\mathbf{u})=\mathbf{0}$ on $\Gamma_{0}$ and $\gamma_{B}^{I}(\mathbf{u}) d s=\boldsymbol{\mu}$ on $\Gamma_{1}$, we have

$$
\begin{aligned}
\left(\mathbb{H}_{\lambda}^{j}\right)^{* *}\left(\varepsilon(\mathbf{u})_{\mid \bar{\Omega}}, \boldsymbol{\mu} \otimes_{s} \boldsymbol{\nu}\right) & =\mathbb{H}_{\lambda}^{j}\left(\varepsilon(\mathbf{u})_{\mid \bar{\Omega}}, \boldsymbol{\mu} \otimes_{s} \boldsymbol{\nu}\right) \\
& =\left(\widetilde{\mathbb{H}}_{\lambda}^{j}\right)^{* *}\left(\varepsilon(\mathbf{u})_{\mid \Omega},-\gamma_{B}^{I}(\mathbf{u}) \otimes_{s} \boldsymbol{\nu}, \boldsymbol{\mu} \otimes_{s} \boldsymbol{\nu}\right) .
\end{aligned}
$$

Proof. By $[1,(4.80)]$, we have $\left(\widetilde{\mathbb{H}}_{\lambda}^{j}\right)^{* *}\left(\varepsilon(\mathbf{u})_{\mid \Omega},-\gamma_{B}^{I}(\mathbf{u}) d s \otimes_{s} \boldsymbol{\nu}, \boldsymbol{\mu} \otimes_{s} \boldsymbol{\nu}\right)=$ $\mathbb{H}_{\lambda}^{j}\left(\varepsilon(\mathbf{u})_{\mid \bar{\Omega}}, \boldsymbol{\mu} \otimes_{s} \boldsymbol{\nu}\right)$ for every $\mathbf{u} \in B D\left(\Omega_{1}\right)$ and $\boldsymbol{\mu} \in \mathbb{M}_{b}\left(\Gamma_{1}, \mathbb{R}^{n}\right)$ such that $\mathbf{u}_{\mid \Omega} \in L D(\Omega), \mathbf{u}_{\mid \Omega_{1}-\bar{\Omega}}=\mathbf{0}, \gamma_{B}^{I}(\mathbf{u})=\mathbf{0}$ on $\Gamma_{0}$ and $\gamma_{B}^{I}(\mathbf{u}) d s=\boldsymbol{\mu}$ on $\Gamma_{1}$ (now $\mathbb{H}^{\# *}$ is denoted by $\left.\left(\widetilde{\mathbb{H}}_{\lambda}^{j}\right)^{* *}\right)$. Similarly to the proofs of Lemmas 6 and 8 from [2] we obtain $(2.15)$.

Lemma 2. Let $\boldsymbol{\kappa}_{s} \in C\left(\Gamma_{1}, \mathbf{E}_{s}^{n}\right), \boldsymbol{\sigma}_{s} \in C_{\operatorname{div}}\left(\bar{\Omega}, \mathbf{E}_{s}^{n}\right)$, where $\operatorname{div} \boldsymbol{\sigma}_{s}=\mathbf{0}$ on $\Omega$. If for every $\mathbf{t} \in L^{1}\left(\Gamma_{1}, \mathbb{R}^{n}\right), \int_{\Gamma_{1}} \boldsymbol{\kappa}_{s}:\left[\mathbf{t} \otimes_{s} \boldsymbol{\nu}\right] d s=0$, then

$$
\left(\mathbb{H}_{\lambda}^{j}\right)^{*}(\boldsymbol{\sigma}, \boldsymbol{\kappa})=\left(\mathbb{H}_{\lambda}^{j}\right)^{*}\left(\boldsymbol{\sigma}+\boldsymbol{\sigma}_{s}, \boldsymbol{\kappa}+\boldsymbol{\kappa}_{s}\right)
$$

for every $\boldsymbol{\sigma} \in C_{\operatorname{div}}\left(\bar{\Omega}, \mathbf{E}_{s}^{n}\right)$ and $\boldsymbol{\kappa} \in C\left(\Gamma_{1}, \mathbf{E}_{s}^{n}\right)$.

Proof. We prove this result similarly to [2, Lemma 9].

We say that a net $\left\{\left(\boldsymbol{\sigma}_{t}, \boldsymbol{\kappa}_{t}\right)\right\}_{t \in T} \subset C_{\operatorname{div}}\left(\bar{\Omega}, \mathbf{E}_{s}^{n}\right) \times C\left(\Gamma_{1}, \mathbf{E}_{s}^{n}\right)$ converges to $(\widehat{\boldsymbol{\sigma}}, \widehat{\boldsymbol{\kappa}})$ in the topology

$$
\begin{aligned}
& \sigma\left(C_{\operatorname{div}}\left(\bar{\Omega}, \mathbf{E}_{s}^{n}\right) \times C\left(\Gamma_{1}, \mathbf{E}_{s}^{n}\right), L^{1}\left(\Omega, \mathbf{E}_{s}^{n}\right) \times\right. \\
& \left.\left\{\left(\boldsymbol{\varphi}, \boldsymbol{\mu} \otimes_{s} \boldsymbol{\nu}\right) \in \mathbf{Y}^{1}(\bar{\Omega})_{\mid \operatorname{Fr} \Omega} \times \mathbf{M}^{1}\left(\Gamma_{1}\right) \mid \boldsymbol{\varphi}_{\mid \Gamma_{0}}=\mathbf{0}, \boldsymbol{\varphi}_{\mid \Gamma_{1}} d s=\boldsymbol{\mu} \otimes_{s} \boldsymbol{\nu}\right\}\right)
\end{aligned}
$$

if $\left\langle\left(\mathbf{w} d x, \mathbf{p} \otimes_{s} \boldsymbol{\nu}, \mathbf{p}_{\mid \Gamma_{1}} d s \otimes_{s} \boldsymbol{\nu}\right) ;\left[\left(\boldsymbol{\sigma}_{t}, \boldsymbol{\kappa}_{t}\right)-(\widehat{\boldsymbol{\sigma}}, \widehat{\boldsymbol{\kappa}})\right]\right\rangle_{3} \rightarrow 0$ for every $\mathbf{w} \in$ $L^{1}\left(\Omega, \mathbf{E}_{s}^{n}\right)$ and $\mathbf{p} \in L^{1}\left(\operatorname{Fr} \Omega, \mathbb{R}^{n}\right)$, where $\mathbf{p}_{\mid \Gamma_{0}}=\mathbf{0}$.

\section{Lemma 3. Let}

$$
\widetilde{f}: C_{\operatorname{div}}\left(\bar{\Omega}, \mathbf{E}_{s}^{n}\right) \times C\left(\Gamma_{1}, \mathbf{E}_{s}^{n}\right) \rightarrow \mathbb{R}
$$

be a linear functional, continuous in the topology (2.16), such that there exist $\boldsymbol{\kappa}_{0} \in C\left(\Gamma_{1}, \mathbf{E}_{s}^{n}\right)$ and $\widetilde{\boldsymbol{\sigma}}_{1} \in C_{\mathrm{div}}\left(\bar{\Omega}, \mathbf{E}_{s}^{n}\right)$ such that for every $\boldsymbol{\sigma}_{s} \in C\left(\bar{\Omega}, \mathbf{E}_{s}^{n}\right)$ 
with $\operatorname{div} \boldsymbol{\sigma}_{s}=\mathbf{0}$, we have

$$
\widetilde{f}\left(\widetilde{\boldsymbol{\sigma}}_{1}+\boldsymbol{\sigma}_{s}, \boldsymbol{\kappa}_{0}\right)=\widetilde{f}\left(\widetilde{\boldsymbol{\sigma}}_{1}, \boldsymbol{\kappa}_{0}\right) .
$$

Moreover, assume that for every $\boldsymbol{\kappa}_{s} \in C\left(\Gamma_{1}, \mathbf{E}_{s}^{n}\right)$ with $\int_{\Gamma_{1}} \boldsymbol{\kappa}_{s}:\left[\mathbf{t} \otimes_{s} \boldsymbol{\nu}\right] d s=0$ for every $\mathbf{t} \in L^{1}\left(\Gamma_{1}, \mathbb{R}^{n}\right)$, we have

$$
\forall \boldsymbol{\sigma} \in C_{\operatorname{div}}\left(\bar{\Omega}, \mathbf{E}_{s}^{n}\right), \forall \boldsymbol{\kappa} \in C\left(\Gamma_{1}, \mathbf{E}_{s}^{n}\right), \quad \tilde{f}(\boldsymbol{\sigma}, \boldsymbol{\kappa})=\widetilde{f}\left(\boldsymbol{\sigma}, \boldsymbol{\kappa}+\boldsymbol{\kappa}_{s}\right) .
$$

Then there exists $\widetilde{\mathbf{u}}_{1} \in L D(\Omega)$ such that $\boldsymbol{\gamma}_{B}\left(\widetilde{\mathbf{u}}_{1}\right)=\mathbf{0}$ on $\Gamma_{0}$ and for every $(\boldsymbol{\sigma}, \boldsymbol{\kappa}) \in C_{\mathrm{div}}\left(\bar{\Omega}, \mathbf{E}_{s}^{n}\right) \times C\left(\Gamma_{1}, \mathbf{E}_{s}^{n}\right)$ we get

$$
\begin{aligned}
\widetilde{f}(\boldsymbol{\sigma}, \boldsymbol{\kappa})= & \int_{\Omega} \boldsymbol{\sigma}: \boldsymbol{\varepsilon}\left(\widetilde{\mathbf{u}}_{1}\right) d x-\int_{\operatorname{Fr} \Omega} \boldsymbol{\sigma}:\left(\boldsymbol{\gamma}_{B}\left(\widetilde{\mathbf{u}}_{1}\right) \otimes_{s} \boldsymbol{\nu}\right) d s \\
& +\int_{\Gamma_{1}} \boldsymbol{\kappa}:\left(\boldsymbol{\gamma}_{B}\left(\widetilde{\mathbf{u}}_{1}\right) \otimes_{s} \boldsymbol{\nu}\right) d s .
\end{aligned}
$$

Proof. Since $\widetilde{f}$ is continuous in the topology (2.16), by Theorem V.3.9 of [8] there exist $\mathbf{m} \in L^{1}\left(\Omega, \mathbf{E}_{s}^{n}\right)$ and $\widehat{\mathbf{u}} \in B D(\Omega)$ with $\gamma_{B}(\widehat{\mathbf{u}})=\mathbf{0}$ on $\Gamma_{0}$ such that, for every $\boldsymbol{\sigma} \in C_{\operatorname{div}}\left(\bar{\Omega}, \mathbf{E}_{s}^{n}\right)$ and $\boldsymbol{\kappa} \in C\left(\Gamma_{1}, \mathbf{E}_{s}^{n}\right)$,

$$
\begin{aligned}
\widetilde{f}(\boldsymbol{\sigma}, \boldsymbol{\kappa})= & \int_{\Omega} \boldsymbol{\sigma}: \mathbf{m} d x-\int_{\operatorname{Fr} \Omega} \boldsymbol{\sigma}:\left(\boldsymbol{\gamma}_{B}(\widehat{\mathbf{u}}) \otimes_{s} \boldsymbol{\nu}\right) d s \\
& +\int_{\Gamma_{1}} \boldsymbol{\kappa}:\left(\boldsymbol{\gamma}_{B}(\widehat{\mathbf{u}}) \otimes_{s} \boldsymbol{\nu}\right) d s .
\end{aligned}
$$

Indeed, in (2.17) we can replace $C\left(\Gamma_{1}, \mathbf{E}_{s}^{n}\right)$ by

$$
C\left(\Gamma_{1}, \mathbf{E}_{s}^{n}\right) /\left\{\boldsymbol{\kappa} \in C\left(\Gamma_{1}, \mathbf{E}_{s}^{n}\right) \mid \forall \mathbf{t} \in L^{1}\left(\Gamma_{1}, \mathbb{R}^{n}\right), \int_{\Gamma_{1}} \boldsymbol{\kappa}:\left[\mathbf{t} \otimes_{s} \boldsymbol{\nu}\right] d s=0\right\} .
$$

By the linearity of $\tilde{f}$, we get $\widetilde{f}\left(\boldsymbol{\sigma}+\boldsymbol{\sigma}_{s}, \boldsymbol{\kappa}\right)=\tilde{f}(\boldsymbol{\sigma}, \boldsymbol{\kappa})$ for every $\boldsymbol{\kappa} \epsilon$ $C\left(\Gamma_{1}, \mathbf{E}_{s}^{n}\right), \boldsymbol{\sigma} \in C_{\operatorname{div}}\left(\bar{\Omega}, \mathbf{E}_{s}^{n}\right)$ and $\boldsymbol{\sigma}_{s} \in C_{\operatorname{div}}\left(\bar{\Omega}, \mathbf{E}_{s}^{n}\right)$ such that $\operatorname{div} \boldsymbol{\sigma}_{s}=\mathbf{0}$. Indeed, by (2.18) and (2.21) we have

$$
\begin{aligned}
\widetilde{f}\left(\boldsymbol{\sigma}+\boldsymbol{\sigma}_{s}, \boldsymbol{\kappa}_{0}\right) & =\widetilde{f}\left(\widetilde{\boldsymbol{\sigma}}_{1}+\boldsymbol{\sigma}_{s}, \boldsymbol{\kappa}_{0}\right)+\widetilde{f}\left(\boldsymbol{\sigma}-\widetilde{\boldsymbol{\sigma}}_{1}, \mathbf{0}\right) \\
& =\widetilde{f}\left(\widetilde{\boldsymbol{\sigma}}_{1}, \boldsymbol{\kappa}_{0}\right)+\widetilde{f}\left(\boldsymbol{\sigma}-\widetilde{\boldsymbol{\sigma}}_{1}, \mathbf{0}\right)=\widetilde{f}\left(\boldsymbol{\sigma}, \boldsymbol{\kappa}_{0}\right),
\end{aligned}
$$

and by $(2.21)$ we get

$$
\begin{aligned}
\widetilde{f}\left(\boldsymbol{\sigma}+\boldsymbol{\sigma}_{s}, \boldsymbol{\kappa}\right) & =\widetilde{f}\left(\boldsymbol{\sigma}+\boldsymbol{\sigma}_{s}, \boldsymbol{\kappa}_{0}\right)+\widetilde{f}\left(\mathbf{0}, \boldsymbol{\kappa}-\boldsymbol{\kappa}_{0}\right) \\
& =\widetilde{f}\left(\boldsymbol{\sigma}, \boldsymbol{\kappa}_{0}\right)+\widetilde{f}\left(\mathbf{0}, \boldsymbol{\kappa}-\boldsymbol{\kappa}_{0}\right)=\widetilde{f}(\boldsymbol{\sigma}, \boldsymbol{\kappa}) .
\end{aligned}
$$

Thus, $\widetilde{f}\left(\boldsymbol{\sigma}_{s}, \mathbf{0}\right)=0$ for every $\boldsymbol{\sigma}_{s} \in C\left(\bar{\Omega}, \mathbf{E}_{s}^{n}\right)$ with $\operatorname{div} \boldsymbol{\sigma}_{s}=\mathbf{0}$. Taking $\boldsymbol{\kappa}=\mathbf{0}$ in (2.21), we may proceed as in [2, Lemma 10].

Let $Q_{1}: C_{\operatorname{div}}\left(\bar{\Omega}, \mathbf{E}_{s}^{n}\right) \times C\left(\Gamma_{1}, \mathbf{E}_{s}^{n}\right) \rightarrow \mathbb{R} \cup\{\infty\}$ be defined by 


$$
\begin{aligned}
& Q_{1}(\boldsymbol{\sigma}, \boldsymbol{\kappa}) \\
& \quad=\inf _{\boldsymbol{\sigma}_{s}}\left\{\left(\widetilde{\mathbb{H}}_{\lambda}^{j}\right)^{*}\left(\boldsymbol{\sigma}+\boldsymbol{\sigma}_{s}, \boldsymbol{\kappa}\right) \mid \boldsymbol{\sigma}_{s} \in C\left(\bar{\Omega}, \mathbf{E}_{s}^{n}\right) \text { with } \operatorname{div} \boldsymbol{\sigma}_{s}=\mathbf{0}\right\}
\end{aligned}
$$

for $\boldsymbol{\sigma} \in C_{\mathrm{div}}\left(\bar{\Omega}, \mathbf{E}_{s}^{n}\right)$ and $\boldsymbol{\kappa} \in C\left(\Gamma_{1}, \mathbf{E}_{s}^{n}\right)$.

Proposition 4. For every $\boldsymbol{\sigma} \in C_{\operatorname{div}}\left(\bar{\Omega}, \mathbf{E}_{s}^{n}\right)$ and $\boldsymbol{\kappa} \in C\left(\Gamma_{1}, \mathbf{E}_{s}^{n}\right)$ we have

$$
\left(\mathbb{H}_{\lambda}^{j}\right)^{*}(\boldsymbol{\sigma}, \boldsymbol{\kappa})=\operatorname{cl}_{(2.16)} Q_{1}(\boldsymbol{\sigma}, \boldsymbol{\kappa}),
$$

where $\mathrm{cl}_{(2.16)} Q_{1}$ denotes the largest minorant which is less than $Q_{1}$ and is l.s.c. in the topology $(2.16)\left(\right.$ i.e. $\operatorname{cl}_{(2.16)} Q_{1}$ is the l.s.c. regularization of $Q_{1}$ in (2.16)).

Proof. Step 1. First notice that

$$
Q_{1}(\boldsymbol{\sigma}, \boldsymbol{\kappa})=\inf _{\boldsymbol{\sigma}_{s}, \boldsymbol{\kappa}_{s}}\left\{\left(\widetilde{\mathbb{H}}_{\lambda}^{j}\right)^{*}\left(\boldsymbol{\sigma}+\boldsymbol{\sigma}_{s}, \boldsymbol{\kappa}+\boldsymbol{\kappa}_{s}\right) \mid \boldsymbol{\sigma}_{s} \in C\left(\bar{\Omega}, \mathbf{E}_{s}^{n}\right),\right.
$$

$$
\left.\boldsymbol{\kappa}_{s} \in C\left(\Gamma_{1}, \mathbf{E}_{s}^{n}\right), \operatorname{div} \boldsymbol{\sigma}_{s}=\mathbf{0} \text { and } \forall \mathbf{t} \in L^{1}\left(\Gamma_{1}, \mathbb{R}^{n}\right), \int_{\Gamma_{1}} \boldsymbol{\kappa}_{s}:\left[\mathbf{t} \otimes_{s} \boldsymbol{\nu}\right] d s=0\right\}
$$

(see $(2.11)-(2.13))$. Next, assume the existence of a couple $\left(\widehat{\boldsymbol{\sigma}}_{1}, \boldsymbol{\kappa}_{0}\right) \in$ $C_{\text {div }}\left(\bar{\Omega}, \mathbf{E}_{s}^{n}\right) \times C\left(\Gamma_{1}, \mathbf{E}_{s}^{n}\right)$ and a constant $\delta_{0}>0$ such that $\left(\mathbb{H}_{\lambda}^{j}\right)^{*}\left(\widehat{\boldsymbol{\sigma}}_{1}, \boldsymbol{\kappa}_{0}\right)+$ $\delta_{0}<\operatorname{cl}_{(2.16)} Q_{1}\left(\widehat{\boldsymbol{\sigma}}_{1}, \boldsymbol{\kappa}_{0}\right)$. On account of Lemma 2, it suffices to show that this assumption leads to a contradiction, since $\left(\mathbb{H}_{\lambda}^{j}\right)^{*}(\boldsymbol{\sigma}, \boldsymbol{\kappa}) \leq\left(\widetilde{\mathbb{H}}_{\lambda}^{j}\right)^{*}(\boldsymbol{\sigma}, \boldsymbol{\kappa})$ for every $(\boldsymbol{\sigma}, \boldsymbol{\kappa}) \in C_{\mathrm{div}}\left(\bar{\Omega}, \mathbf{E}_{s}^{n}\right) \times C\left(\Gamma_{1}, \mathbf{E}_{s}^{n}\right)$ (cf. (2.7)-(2.9), (2.11)-(2.13)).

Step 2. The linear space

$$
\begin{aligned}
\mathcal{M}_{1} \equiv & \left\{\boldsymbol{\sigma}_{s} \in C_{\operatorname{div}}\left(\bar{\Omega}, \mathbf{E}_{s}^{n}\right) \mid \operatorname{div} \boldsymbol{\sigma}_{s}=\mathbf{0}\right\} \\
& \times\left\{\boldsymbol{\kappa} \in C\left(\Gamma_{1}, \mathbf{E}_{s}^{n}\right) \mid \forall \mathbf{t} \in L^{1}\left(\Gamma_{1}, \mathbb{R}^{n}\right), \int_{\Gamma_{1}} \boldsymbol{\kappa}:\left[\mathbf{t} \otimes_{s} \boldsymbol{\nu}\right] d s=0\right\}
\end{aligned}
$$

is a closed subspace of $C_{\text {div }}\left(\bar{\Omega}, \mathbf{E}_{s}^{n}\right) \times C\left(\Gamma_{1}, \mathbf{E}_{s}^{n}\right)$ endowed with the topology (2.16). Indeed, by Green's formula (see [2, (2.11) and (4.23)]) we have

$$
\begin{array}{r}
\bigcap_{\mathbf{u} \in L D(\Omega), \gamma_{B}(\mathbf{u})=\mathbf{0} \text { on } \Gamma_{0}}\left\{(\boldsymbol{\sigma}, \boldsymbol{\kappa}) \in C_{\operatorname{div}}\left(\bar{\Omega}, \mathbf{E}_{s}^{n}\right) \times C\left(\Gamma_{1}, \mathbf{E}_{s}^{n}\right) \mid\right. \\
\left.\int_{\Omega}(\operatorname{div} \boldsymbol{\sigma}) \cdot \mathbf{u} d x=\int_{\Gamma_{1}} \boldsymbol{\kappa}:\left(\gamma_{B}(\mathbf{u}) \otimes_{s} \boldsymbol{\nu}\right) d s\right\} \\
=\bigcap_{\mathbf{u} \in L D(\Omega), \gamma_{B}(\mathbf{u})=\mathbf{0} \text { on } \Gamma_{0}}\left\{(\boldsymbol{\sigma}, \boldsymbol{\kappa}) \in C_{\operatorname{div}}\left(\bar{\Omega}, \mathbf{E}_{s}^{n}\right) \times C\left(\Gamma_{1}, \mathbf{E}_{s}^{n}\right) \mid\right. \\
\left.\operatorname{div} \boldsymbol{\sigma}=\mathbf{0} \text { in } \Omega, \int_{\Gamma_{1}} \boldsymbol{\kappa}:\left(\boldsymbol{\gamma}_{B}(\mathbf{u}) \otimes_{s} \boldsymbol{\nu}\right) d s=0\right\} \\
=\left\{(\boldsymbol{\sigma}, \boldsymbol{\kappa}) \mid \operatorname{div} \boldsymbol{\sigma}=\mathbf{0}, \int_{\Gamma_{1}} \boldsymbol{\kappa}:\left[\mathbf{t} \otimes_{s} \boldsymbol{\nu}\right] d s=0, \forall \mathbf{t} \in L^{1}\left(\Gamma_{1}, \mathbb{R}^{n}\right)\right\}=\mathcal{M}_{1},
\end{array}
$$

since the trace $\gamma_{B}$ is a surjection onto $L^{1}\left(\operatorname{Fr} \Omega, \mathbb{R}^{n}\right)$. 
Step 3. Let

$$
\Phi_{1}: C_{\mathrm{div}}\left(\bar{\Omega}, \mathbf{E}_{s}^{n}\right) \times C\left(\Gamma_{1}, \mathbf{E}_{s}^{n}\right) \rightarrow\left[C_{\mathrm{div}}\left(\bar{\Omega}, \mathbf{E}_{s}^{n}\right) \times C\left(\Gamma_{1}, \mathbf{E}_{s}^{n}\right)\right] / \mathcal{M}_{1}
$$

be the linear function (canonical homomorphism) such that $\mathcal{M}_{1}=\operatorname{ker} \Phi_{1}$. Moreover, let $\left[C_{\text {div }}\left(\bar{\Omega}, \mathbf{E}_{s}^{n}\right) \times C\left(\Gamma_{1}, \mathbf{E}_{s}^{n}\right)\right] / \mathcal{M}_{1}$ be endowed with the strongest topology for which $\Phi_{1}$ is continuous, where $C_{\operatorname{div}}\left(\bar{\Omega}, \mathbf{E}_{s}^{n}\right) \times C\left(\Gamma_{1}, \mathbf{E}_{s}^{n}\right)$ is endowed with the topology (2.16). Then

$$
\begin{aligned}
& \widehat{A}_{1} \equiv\left\{((\widetilde{\boldsymbol{\sigma}}, \widetilde{\boldsymbol{\kappa}}), a) \in\left[\left(C_{\mathrm{div}}\left(\bar{\Omega}, \mathbf{E}_{s}^{n}\right) \times C\left(\Gamma_{1}, \mathbf{E}_{s}^{n}\right)\right) / \mathcal{M}_{1}\right] \times \mathbb{R} \mid\right. \\
& \left.\quad \exists(\boldsymbol{\sigma}, \boldsymbol{\kappa}) \text { such that } \operatorname{cl}_{(2.16)} Q_{1}(\boldsymbol{\sigma}, \boldsymbol{\kappa}) \leq a \text { and } \Phi_{1}(\boldsymbol{\sigma}, \boldsymbol{\kappa})=(\widetilde{\boldsymbol{\sigma}}, \widetilde{\boldsymbol{\kappa}})\right\}
\end{aligned}
$$

is a convex closed set. By the Hahn-Banach theorem, there exists a closed affine hyperplane $\mathcal{H}_{1}$ which strictly separates the set $\widehat{A}_{1}$ and $\left(\Phi_{1}\left(\widehat{\boldsymbol{\sigma}}_{1}, \boldsymbol{\kappa}_{0}\right)\right.$, $\left.\left(\mathbb{H}_{\lambda}^{j}\right)^{*}\left(\widehat{\boldsymbol{\sigma}}_{1}, \boldsymbol{\kappa}_{0}\right)+\delta_{0}\right)$. From [1, (4.79)] and Assumption 4, we deduce that $\inf \left\{\left(\widetilde{\mathbb{H}}_{\lambda}^{j}\right)^{*}(\boldsymbol{\sigma}, \boldsymbol{\kappa})-\int_{\Omega} \boldsymbol{\varepsilon}\left(\mathbf{u}^{e}\right): \boldsymbol{\sigma} d x \mid \boldsymbol{\sigma} \in C_{\mathrm{div}}\left(\bar{\Omega}, \mathbf{E}_{s}^{n}\right), \boldsymbol{\kappa} \in C\left(\Gamma_{1}, \mathbf{E}_{s}^{n}\right)\right\}$ is finite (here $\mathbb{H}^{\#}$ is denoted by $\left.\left(\widetilde{\mathbb{H}}_{\lambda}^{j}\right)^{*}\right)$. Then, similarly to Step 4 of the proof of Proposition 11 from [2], there exists a continuous linear functional $\widetilde{f}_{3}$ : $\left[C_{\mathrm{div}}\left(\bar{\Omega}, \mathbf{E}_{s}^{n}\right) \times C\left(\Gamma_{1}, \mathbf{E}_{s}^{n}\right)\right] / \mathcal{M}_{1} \rightarrow \mathbb{R}$ and $\widetilde{c}_{3} \in \mathbb{R}$ such that

$$
\widetilde{f}_{3}\left(\Phi_{1}\left(\widehat{\boldsymbol{\sigma}}_{1}, \boldsymbol{\kappa}_{0}\right)\right)+\widetilde{c}_{3}>\left(\mathbb{H}_{\lambda}^{j}\right)^{*}\left(\widehat{\boldsymbol{\sigma}}_{1}, \boldsymbol{\kappa}_{0}\right)+\delta_{0}, \quad f_{3}(\widetilde{\boldsymbol{\sigma}}, \widetilde{\boldsymbol{\kappa}})+c_{3}<a
$$

for every $((\tilde{\boldsymbol{\sigma}}, \widetilde{\boldsymbol{\kappa}}), a) \in \widehat{A}_{1}$.

Step 4 . Therefore the linear functional $\widetilde{f}_{4}$ defined by $\widetilde{f}_{4}=\widetilde{f}_{3} \circ \Phi_{1}$ strictly separates epi $\operatorname{cl}_{(2.16)} Q_{1}$ and

$$
\begin{aligned}
&\left\{(\boldsymbol{\sigma}, \boldsymbol{\kappa}, a) \in C_{\mathrm{div}}\left(\bar{\Omega}, \mathbf{E}_{s}^{n}\right) \times C\left(\Gamma_{1}, \mathbf{E}_{s}^{n}\right) \times \mathbb{R} \mid\right. \\
&\left.(\boldsymbol{\sigma}, \boldsymbol{\kappa}) \in \mathcal{M}_{1}+\left\{\left(\widehat{\boldsymbol{\sigma}}_{1}, \boldsymbol{\kappa}_{0}\right)\right\}, a=\left(\mathbb{H}_{\lambda}^{j}\right)^{*}\left(\widehat{\boldsymbol{\sigma}}_{1}, \boldsymbol{\kappa}_{0}\right)+\delta_{0}\right\} .
\end{aligned}
$$

Moreover $\mathcal{M}_{1} \subset \operatorname{ker} \widetilde{f}_{4}$ and $\widetilde{f}_{4}$ is continuous in the topology (2.16), since $\tilde{f}_{4}=\tilde{f}_{3} \circ \Phi_{1}$.

Step 5. By Lemma 3, there exists $\widetilde{\mathbf{u}}_{1} \in L D(\Omega)$ such that $\boldsymbol{\gamma}_{B}\left(\widetilde{\mathbf{u}}_{1}\right)=\mathbf{0}$ on $\Gamma_{0}$ and for every $(\boldsymbol{\sigma}, \boldsymbol{\kappa}) \in C_{\operatorname{div}}\left(\bar{\Omega}, \mathbf{E}_{s}^{n}\right) \times C\left(\Gamma_{1}, \mathbf{E}_{s}^{n}\right)$,

$$
\begin{aligned}
\widetilde{f}_{4}(\boldsymbol{\sigma}, \boldsymbol{\kappa})= & \int_{\Omega} \boldsymbol{\sigma}: \boldsymbol{\varepsilon}\left(\widetilde{\mathbf{u}}_{1}\right) d x-\int_{\operatorname{Fr} \Omega} \boldsymbol{\sigma}:\left(\boldsymbol{\gamma}_{B}\left(\widetilde{\mathbf{u}}_{1}\right) \otimes_{s} \boldsymbol{\nu}\right) d s \\
& +\int_{\Gamma_{1}} \boldsymbol{\kappa}:\left(\boldsymbol{\gamma}_{B}\left(\widetilde{\mathbf{u}}_{1}\right) \otimes_{s} \boldsymbol{\nu}\right) d s .
\end{aligned}
$$

Step 6. We say that a net $\left\{\left(\boldsymbol{\sigma}_{t}, \boldsymbol{\kappa}_{t}\right)\right\}_{t \in T} \subset C_{\mathrm{div}}\left(\bar{\Omega}, \mathbf{E}_{s}^{n}\right) \times C\left(\Gamma_{1}, \mathbf{E}_{s}^{n}\right)$ converges to $(\widehat{\boldsymbol{\sigma}}, \widehat{\boldsymbol{\kappa}})$ in the topology

$$
\begin{array}{r}
\sigma\left(C_{\mathrm{div}}\left(\bar{\Omega}, \mathbf{E}_{s}^{n}\right) \times C\left(\Gamma_{1}, \mathbf{E}_{s}^{n}\right) ;\left\{\left(\boldsymbol{\varphi}, \boldsymbol{\mu} \otimes_{s} \boldsymbol{\nu}\right) \in \mathbf{Y}^{1}(\bar{\Omega}) \times \mathbf{M}^{1}\left(\Gamma_{1}\right) \mid\right.\right. \\
\exists \mathbf{u} \in B D\left(\Omega_{1}\right), \boldsymbol{\varepsilon}(\mathbf{u})=\boldsymbol{\varphi}, \mathbf{u}_{\mid \Omega} \in L D(\Omega), \mathbf{u}_{\mid \Omega_{1}-\bar{\Omega}}=\mathbf{0}, \\
\left.\left.\gamma_{B}^{I}(\mathbf{u})=\mathbf{0} \text { on } \Gamma_{0} \text { and } \gamma_{B}^{I}(\mathbf{u}) d s=\boldsymbol{\mu} \text { on } \Gamma_{1}\right\}\right)
\end{array}
$$


if

$$
\left\langle\left(\varepsilon(\mathbf{u})_{\mid \bar{\Omega}}, \gamma_{B}^{I}(\mathbf{u}) d s \otimes_{s} \boldsymbol{\nu}\right) ;\left(\boldsymbol{\sigma}_{t}, \boldsymbol{\kappa}_{t}\right)-(\widehat{\boldsymbol{\sigma}}, \widehat{\boldsymbol{\kappa}})\right\rangle_{2} \rightarrow 0
$$

for every $\mathbf{u} \in B D\left(\Omega_{1}\right)$, where $\mathbf{u}_{\mid \Omega} \in L D(\Omega), \mathbf{u}_{\mid \Omega_{1}-\bar{\Omega}}=\mathbf{0}$ and $\gamma_{B}^{I}(\mathbf{u})=\mathbf{0}$ on $\Gamma_{0}$. The l.s.c. regularization of $\left(\widetilde{\mathbb{H}}_{\lambda}^{j}\right)^{*}$ in the topology $(2.35)$ (denoted by $\left.\operatorname{cl}_{(2.35)}\left(\widetilde{\mathbb{H}}_{\lambda}^{j}\right)^{*}\right)$ is given by

$$
\begin{array}{r}
\operatorname{cl}_{(2.35)}\left(\widetilde{\mathbb{H}}_{\lambda}^{j}\right)^{*}(\boldsymbol{\sigma}, \boldsymbol{\kappa})=\sup \left\{\left\langle\left(\varepsilon(\mathbf{u})_{\mid \bar{\Omega}}, \gamma_{B}^{I}(\mathbf{u}) d s \otimes_{s} \boldsymbol{\nu}\right) ;(\boldsymbol{\sigma}, \boldsymbol{\kappa})\right\rangle_{2}\right. \\
-\left(\widetilde{\mathbb{H}}_{\lambda}^{j}\right)^{* *}\left(\varepsilon(\mathbf{u})_{\mid \bar{\Omega}}, \gamma_{B}^{I}(\mathbf{u}) d s \otimes_{s} \boldsymbol{\nu}\right) \mid \mathbf{u} \in B D\left(\Omega_{1}\right), \mathbf{u}_{\mid \Omega} \in L D(\Omega), \\
\left.\mathbf{u}_{\mid \Omega_{1}-\bar{\Omega}}=\mathbf{0} \text { and } \boldsymbol{\gamma}_{B}^{I}(\mathbf{u})=\mathbf{0} \text { on } \Gamma_{0}\right\}=\sup \left\{\left\langle\varepsilon(\mathbf{u})_{\mid \bar{\Omega}},\left(\boldsymbol{\gamma}_{B}^{I}(\mathbf{u}) d s \otimes_{s} \boldsymbol{\nu}\right)\right.\right. \\
(\boldsymbol{\sigma}, \boldsymbol{\kappa})\rangle_{2}-\mathbb{H}_{\lambda}^{j}\left(\boldsymbol{\varepsilon}(\mathbf{u})_{\mid \bar{\Omega}}, \gamma_{B}^{I}(\mathbf{u}) d s\right) \mid \mathbf{u} \in B D\left(\Omega_{1}\right), \\
\left.\mathbf{u}_{\mid \Omega} \in L D(\Omega), \mathbf{u}_{\mid \Omega_{1}-\bar{\Omega}}=\mathbf{0} \text { and } \gamma_{B}^{I}(\mathbf{u})=\mathbf{0} \text { on } \Gamma_{0}\right\}=\left(\mathbb{H}_{\lambda}^{j}\right)^{*}(\boldsymbol{\sigma}, \boldsymbol{\kappa}),
\end{array}
$$

for every $\boldsymbol{\sigma} \in C_{\operatorname{div}}\left(\bar{\Omega}, \mathbf{E}_{s}^{n}\right)$ and $\boldsymbol{\kappa} \in C\left(\Gamma_{1}, \mathbf{E}_{s}^{n}\right)$ (see Lemma 1 ).

By $(2.32),(2.34)$ and $(2.37)$ we obtain a contradiction, because $\widetilde{f}_{4}$ is continuous in $(2.35)$.

We say that a net $\left\{\left(\boldsymbol{\sigma}_{t}, \boldsymbol{\kappa}_{t}\right)\right\}_{t \in T} \subset C_{\text {div }}\left(\bar{\Omega}, \mathbf{E}_{s}^{n}\right) \times C\left(\Gamma_{1}, \mathbf{E}_{s}^{n}\right)$ converges to $(\overline{\boldsymbol{\sigma}}, \overline{\boldsymbol{\kappa}})$ in the topology

$$
\sigma\left(C_{\operatorname{div}}\left(\bar{\Omega}, \mathbf{E}_{s}^{n}\right) \times C\left(\Gamma_{1}, \mathbf{E}_{s}^{n}\right), \mathbf{Y}^{1}(\bar{\Omega}) \times L^{1}\left(\Gamma_{1}, \mathbb{R}^{n}\right) \otimes_{s} \boldsymbol{\nu}\right)
$$

if

$$
\left\langle\left(\varepsilon(\mathbf{u})_{\mid \bar{\Omega}}, \mathbf{z} d s \otimes_{s} \boldsymbol{\nu}\right) ;\left(\boldsymbol{\sigma}_{t}, \boldsymbol{\kappa}_{t}\right)-(\overline{\boldsymbol{\sigma}}, \overline{\boldsymbol{\kappa}})\right\rangle_{2} \rightarrow 0
$$

for every $\boldsymbol{\varepsilon}(\mathbf{u})_{\mid \bar{\Omega}} \in \mathbf{Y}^{1}(\bar{\Omega})$ and $\mathbf{z} \in L^{1}\left(\Gamma_{1}, \mathbb{R}^{n}\right)$.

LEMMA 5. For every $k>0$, the topology (2.16) is stronger than (2.38) over the set $\bar{A}_{k} \equiv\left\{(\boldsymbol{\sigma}, \boldsymbol{\kappa}) \in C_{\operatorname{div}}\left(\bar{\Omega}, \mathbf{E}_{s}^{n}\right) \times C\left(\Gamma_{1}, \mathbf{E}_{s}^{n}\right) \mid\|\operatorname{div} \boldsymbol{\sigma}\|_{L^{n}} \leq k\right.$, $\left.\|\boldsymbol{\kappa}\|_{C\left(\Gamma_{1}, \mathbf{E}_{s}^{n}\right)} \leq k\right\}$.

Proof. Suppose a net $\left\{\left(\boldsymbol{\sigma}_{t}, \boldsymbol{\kappa}_{t}\right)\right\}_{t \in T} \subset \bar{A}_{k}$ converges to $(\overline{\boldsymbol{\sigma}}, \overline{\boldsymbol{\kappa}})$ in the topology (2.16). Then by the Green formula (see [2]) we obtain $\int_{\Gamma_{1}}\left(\boldsymbol{\kappa}_{t}-\overline{\boldsymbol{\kappa}}\right)$ : $\left(\boldsymbol{\gamma}_{B}(\mathbf{u}) \otimes_{s} \boldsymbol{\nu}\right) d s-\int_{\Omega} \operatorname{div}\left(\boldsymbol{\sigma}_{t}-\overline{\boldsymbol{\sigma}}\right) \cdot \mathbf{u} d x \rightarrow 0$ for every $\mathbf{u} \in L D(\Omega)$ such that $\gamma_{B}(\mathbf{u})=\mathbf{0}$ on $\Gamma_{0}$. Since $C_{c}^{1}\left(\Omega, \mathbb{R}^{n}\right)$ is dense in $L^{n /(n-1)}\left(\Omega, \mathbb{R}^{n}\right)$, so is $C_{c}^{1}\left(\Omega, \mathbb{R}^{n}\right)+\{\mathbf{u}\}$ for every $\mathbf{u} \in L D(\Omega)$. Therefore the set

$$
\begin{gathered}
\left\{\left(\mathbf{w}, \mathbf{z} \otimes_{s} \boldsymbol{\nu}\right) \in L^{n /(n-1)}\left(\Omega, \mathbb{R}^{n}\right) \times\left(L^{1}\left(\Gamma_{1}, \mathbb{R}^{n}\right) \otimes_{s} \boldsymbol{\nu}\right) \mid\right. \\
\left.\exists \widehat{\mathbf{u}}_{1} \in C_{c}^{1}\left(\Omega, \mathbb{R}^{n}\right), \exists \widehat{\mathbf{u}}_{2} \in L D(\Omega), \mathbf{w}=\widehat{\mathbf{u}}_{1}+\widehat{\mathbf{u}}_{2}, \gamma_{B}\left(\widehat{\mathbf{u}}_{2}\right)=\mathbf{z} \text { on } \Gamma_{1}\right\}
\end{gathered}
$$

is dense in $L^{n /(n-1)}\left(\Omega, \mathbb{R}^{n}\right) \times\left(L^{1}\left(\Gamma_{1}, \mathbb{R}^{n}\right) \otimes_{s} \boldsymbol{\nu}\right)$. By [8, Theorem II.1.18],

$$
\int_{\Gamma_{1}}\left(\boldsymbol{\kappa}_{t}-\overline{\boldsymbol{\kappa}}\right):\left(\mathbf{z} \otimes_{s} \boldsymbol{\nu}\right) d s-\int_{\Omega} \operatorname{div}\left(\boldsymbol{\sigma}_{t}-\overline{\boldsymbol{\sigma}}\right) \cdot \mathbf{u} d x \rightarrow 0
$$


for all $\mathbf{u} \in B D(\Omega)$ and $\mathbf{z} \in L^{1}\left(\Gamma_{1}, \mathbb{R}^{n}\right)$. By the Green formula, $\left\{\left(\boldsymbol{\sigma}_{t}, \boldsymbol{\kappa}_{t}\right)\right\}_{t \in T}$ converges to $(\overline{\boldsymbol{\sigma}}, \overline{\boldsymbol{\kappa}})$ in the topology $(2.38)$.

Proposition 6. Let $\bar{A}_{k}$ be as in Lemma 5. For every $\widehat{\boldsymbol{\sigma}} \in C_{\mathrm{div}}\left(\bar{\Omega}, \mathbf{E}_{s}^{n}\right)$ and $\widehat{\boldsymbol{\kappa}} \in C\left(\Gamma_{1}, \mathbf{E}_{s}^{n}\right)$ there exists $k_{(\widehat{\boldsymbol{\sigma}}, \widehat{\boldsymbol{\kappa}})}>0$ such that for every $k \geq k_{(\widehat{\boldsymbol{\sigma}}, \widehat{\boldsymbol{\kappa}})}$,

$$
\left(\mathbb{H}_{\lambda}^{j}\right)^{*}(\widehat{\boldsymbol{\sigma}}, \widehat{\boldsymbol{\kappa}})=\operatorname{cl}_{\bar{A}_{k}} Q_{1}(\widehat{\boldsymbol{\sigma}}, \widehat{\boldsymbol{\kappa}}),
$$

where $\operatorname{cl}_{\bar{A}_{k}} Q_{1}(\cdot, \cdot)$ is the l.s.c. regularization of $(\boldsymbol{\sigma}, \boldsymbol{\kappa}) \longmapsto Q_{1}(\boldsymbol{\sigma}, \boldsymbol{\kappa})+I_{\bar{A}_{k}}(\boldsymbol{\sigma}, \boldsymbol{\kappa})$ in the topology (2.16) and $I_{\bar{A}_{k}}(\cdot)$ is the indicator function of $\bar{A}_{k}$.

Proof. We prove this result similarly to [2, Proposition 13].

Theorem 7. For every $\varepsilon(\mathbf{u})_{\mid \bar{\Omega}} \in \mathbf{Y}^{1}(\bar{\Omega})$ and $\boldsymbol{\mu} \in \mathbb{M}_{b}\left(\Gamma_{1}, \mathbb{R}^{n}\right)$ we have

$$
\left(\widetilde{\mathbb{H}}_{\lambda}^{j}\right)^{* *}\left(\varepsilon(\mathbf{u})_{\mid \bar{\Omega}}, \boldsymbol{\mu} \otimes_{s} \boldsymbol{\nu}\right)=\left(\mathbb{H}_{\lambda}^{j}\right)^{* *}\left(\varepsilon(\mathbf{u})_{\mid \bar{\Omega}}, \boldsymbol{\mu} \otimes_{s} \boldsymbol{\nu}\right) .
$$

Proof. Step 1. By Proposition 4, Lemma 5 and Proposition 6 (similarly to the proof of Theorem 14 from [2]) we get $\left(\widetilde{\mathbb{H}}_{\lambda}^{j}\right)^{* *}\left(\varepsilon(\mathbf{u})_{\mid \bar{\Omega}}, \mathbf{z} \otimes_{s} \boldsymbol{\nu}\right)=$ $\left(\mathbb{H}_{\lambda}^{j}\right)^{* *}\left(\varepsilon(\mathbf{u})_{\mid \bar{\Omega}}, \mathbf{z} \otimes_{s} \boldsymbol{\nu}\right)$ for every $\boldsymbol{\varepsilon}(\mathbf{u})_{\mid \bar{\Omega}} \in \mathbf{Y}^{1}(\bar{\Omega})$ and $\mathbf{z} \in L^{1}\left(\Gamma_{1}, \mathbb{R}^{n}\right)$.

Step 2. In $[1,(4.80)]$ we obtain the explicit representation of $\left(\widetilde{\mathbb{H}}_{\lambda}^{j}\right)^{* *}$ :

$$
\begin{aligned}
\left(\widetilde{\mathbb{H}}_{\lambda}^{j}\right)^{* *} & \left(\varepsilon(\mathbf{u})_{\mid \bar{\Omega}}, \boldsymbol{\mu} \otimes_{s} \boldsymbol{\nu}\right)=-\int_{\Gamma_{1}} \lambda \mathbf{g} \boldsymbol{\mu}+\int_{\Omega} j\left(x, \varepsilon(\mathbf{u})_{a}\right) d x \\
& +\int_{\Gamma_{1}} j_{\infty}\left(x, \frac{d\left(\left(\boldsymbol{\mu}-\gamma_{B}^{I}(\mathbf{u}) d s\right) \otimes_{s} \boldsymbol{\nu}\right)}{d\left|\left(\boldsymbol{\mu}-\gamma_{B}^{I}(\mathbf{u}) d s\right) \otimes_{s} \boldsymbol{\nu}\right|}\right) d\left|\left(\boldsymbol{\mu}-\gamma_{B}^{I}(\mathbf{u}) d s\right) \otimes_{s} \boldsymbol{\nu}\right| \\
& +\int_{\Omega} j_{\infty}\left(x, \frac{d \varepsilon(\mathbf{u})_{s}}{d\left|\varepsilon(\mathbf{u})_{s}\right|}\right) d\left|\varepsilon(\mathbf{u})_{s}\right|+\int_{\Gamma_{0}} j_{\infty}\left(x,\left(-\gamma_{B}^{I}(\mathbf{u})\right) \otimes_{s} \boldsymbol{\nu}\right) d s
\end{aligned}
$$

for every $\boldsymbol{\varepsilon}(\mathbf{u})_{\mid \bar{\Omega}} \in \mathbf{Y}^{1}(\bar{\Omega})$ and $\boldsymbol{\mu} \in \mathbb{M}_{b}\left(\Gamma_{1}, \mathbb{R}^{n}\right.$ ) (now $\mathbb{H}^{\# *}$ is denoted by $\left.\left(\widetilde{\mathbb{H}}_{\lambda}^{j}\right)^{* *}\right)$. Consider the function $Q_{\varepsilon(\mathbf{u})_{\mid \bar{\Omega}}}: \mathbf{M}^{1}\left(\Gamma_{1}\right) \rightarrow \mathbb{R} \cup\{\infty\}$ for $\varepsilon(\mathbf{u})_{\mid \bar{\Omega}} \in$ $\mathbf{Y}^{1}(\bar{\Omega})$, defined by

$$
\boldsymbol{\mu} \otimes_{s} \boldsymbol{\nu} \mapsto Q_{\varepsilon(\mathbf{u})_{\mid \bar{\Omega}}}\left(\boldsymbol{\mu} \otimes_{s} \boldsymbol{\nu}\right)=\left\{\begin{array}{cl}
\left(\widetilde{\mathbb{H}}_{\lambda}^{j}\right)^{* *}\left(\varepsilon(\mathbf{u})_{\mid \bar{\Omega}}, \boldsymbol{\mu} \otimes_{s} \boldsymbol{\nu}\right) \\
& \text { if } \boldsymbol{\mu} \in L^{1}\left(\Gamma_{1}, \mathbb{R}^{n}\right), \\
\infty & \text { otherwise. }
\end{array}\right.
$$

By (2.44),

$$
\begin{aligned}
& Q_{\varepsilon(\mathbf{u})_{\mid \bar{\Omega}}}\left(\boldsymbol{\mu} \otimes_{s} \boldsymbol{\nu}\right)=-\int_{\Gamma_{1}} \lambda g \boldsymbol{\mu}+c_{\varepsilon(\mathbf{u})_{\mid \bar{\Omega}}} \\
& \quad+\int_{\Gamma_{1}} j_{\infty}\left(x, \frac{d\left(\left(\boldsymbol{\mu}-\gamma_{B}^{I}(\mathbf{u}) d s\right) \otimes_{s} \boldsymbol{\nu}\right)}{d\left|\left(\boldsymbol{\mu}-\gamma_{B}^{I}(\mathbf{u}) d s\right) \otimes_{s} \boldsymbol{\nu}\right|}\right) d\left|\left(\boldsymbol{\mu}-\gamma_{B}^{I}(\mathbf{u}) d s\right) \otimes_{s} \boldsymbol{\nu}\right|
\end{aligned}
$$


for every $\boldsymbol{\mu} \in L^{1}\left(\Gamma_{1}, \mathbb{R}^{n}\right)$, where $c_{\varepsilon(\mathbf{u})_{\mid \bar{\Omega}}}$ depends only on $\boldsymbol{\varepsilon}(\mathbf{u})_{\mid \bar{\Omega}} \in \mathbf{Y}^{1}(\bar{\Omega})$. We now prove that the l.s.c. regularization of $Q_{\varepsilon(\mathbf{u})_{\mid \bar{\Omega}}}$ in the topology $\sigma\left(\mathbf{M}^{1}\left(\Gamma_{1}\right), C\left(\Gamma_{1}, \mathbf{E}_{s}^{n}\right)\right)$ (denoted by $\left.\operatorname{cl} Q_{\varepsilon(\mathbf{u})_{\mid \bar{\Omega}}}\right)$ is given by

$$
\operatorname{cl} Q_{\varepsilon(\mathbf{u})_{\mid \bar{\Omega}}}\left(\boldsymbol{\mu} \otimes_{s} \boldsymbol{\nu}\right)=\left(\widetilde{\mathbb{H}}_{\lambda}^{j}\right)^{* *}\left(\varepsilon(\mathbf{u})_{\mid \bar{\Omega}}, \boldsymbol{\mu} \otimes_{s} \boldsymbol{\nu}\right)
$$

for $\varepsilon(\mathbf{u})_{\mid \bar{\Omega}} \in \mathbf{Y}^{1}(\bar{\Omega})$ and $\boldsymbol{\mu} \in \mathbb{M}_{b}\left(\Gamma_{1}, \mathbb{R}^{n}\right)$.

Indeed, by [5], we obtain

$$
\begin{aligned}
Q_{\varepsilon(\mathbf{u})_{\mid \bar{\Omega}}}^{*}(\boldsymbol{\kappa}) & \sup \left\{\int_{\Gamma_{1}}(\boldsymbol{\kappa} \cdot \boldsymbol{\nu}+\lambda \mathbf{g}) \cdot \boldsymbol{\mu} d s\right. \\
& \left.-\int_{\Gamma_{1}} j_{\infty}\left(x,\left(\boldsymbol{\mu}-\gamma_{B}^{I}(\mathbf{u})\right) \otimes_{s} \boldsymbol{\nu}\right) d s-c_{\varepsilon(\mathbf{u})_{\mid \bar{\Omega}}} \mid \boldsymbol{\mu} \in L^{1}\left(\Gamma_{1}, \mathbb{R}^{n}\right)\right\}
\end{aligned}
$$$$
=\sup \left\{\int_{\Gamma_{1}}(\boldsymbol{\kappa} \cdot \boldsymbol{\nu}+\lambda \mathbf{g}) \cdot\left(\boldsymbol{\mu}-\gamma_{B}^{I}(\mathbf{u})\right) d s-\int_{\Gamma_{1}} j_{\infty}\left(x,\left(\boldsymbol{\mu}-\gamma_{B}^{I}(\mathbf{u})\right) \otimes_{s} \boldsymbol{\nu}\right) d s\right.
$$$$
\left.-c_{\varepsilon(\mathbf{u})_{\mid \bar{\Omega}}} \mid \boldsymbol{\mu} \in L^{1}\left(\Gamma_{1}, \mathbb{R}^{n}\right)\right\}+\int_{\Gamma_{1}}(\boldsymbol{\kappa} \cdot \boldsymbol{\nu}+\lambda \mathbf{g}) \cdot \gamma_{B}^{I}(\mathbf{u}) d s
$$

$=\int_{\Gamma_{1}}(\boldsymbol{\kappa} \cdot \boldsymbol{\nu}+\lambda \mathbf{g}) \cdot \boldsymbol{\gamma}_{B}^{I}(\mathbf{u}) d s+\int_{\Gamma_{1}} I_{\{\boldsymbol{\kappa} \cdot \boldsymbol{\nu}+\lambda \mathbf{g} \in \mathcal{K}(x) \cdot \boldsymbol{\nu}(x)\}}(\boldsymbol{\kappa} \cdot \boldsymbol{\nu}+\lambda \mathbf{g}) d s-c_{\varepsilon(\mathbf{u})_{\mid \bar{\Omega}}}$

for every $\boldsymbol{\kappa} \in C\left(\Gamma_{1}, \mathbf{E}_{s}^{n}\right)$, and

$$
\begin{gathered}
Q_{\varepsilon(\mathbf{u})_{\mid \bar{\Omega}}}^{* *}\left(\boldsymbol{\mu} \otimes_{s} \boldsymbol{\nu}\right)=\sup \left\{\int_{\Gamma_{1}} \boldsymbol{\kappa}:\left(\boldsymbol{\mu} \otimes_{s} \boldsymbol{\nu}\right)-\int_{\Gamma_{1}}(\boldsymbol{\kappa} \cdot \boldsymbol{\nu}+\lambda \mathbf{g}) \cdot \gamma_{B}^{I}(\mathbf{u}) d s\right. \\
\left.-\int_{\Gamma_{1}} I_{\{\boldsymbol{\kappa} \cdot \boldsymbol{\nu}+\lambda \mathbf{g} \in \mathcal{K}(x) \cdot \boldsymbol{\nu}(x)\}}(\boldsymbol{\kappa} \cdot \boldsymbol{\nu}+\lambda \mathbf{g}) d s+c_{\varepsilon(\mathbf{u})_{\mid \bar{\Omega}}} \mid \boldsymbol{\kappa} \in C\left(\Gamma_{1}, \mathbf{E}_{s}^{n}\right)\right\}
\end{gathered}
$$

$=\sup \left\{\int_{\Gamma_{1}}(\boldsymbol{\kappa} \cdot \boldsymbol{\nu}+\lambda \mathbf{g}) \cdot\left(\boldsymbol{\mu}-\boldsymbol{\gamma}_{B}^{I}(\mathbf{u}) d s\right)-\int_{\Gamma_{1}} I_{\{\boldsymbol{\kappa} \cdot \boldsymbol{\nu}+\lambda \mathbf{g} \in \mathcal{K}(x) \cdot \boldsymbol{\nu}(x)\}}(\boldsymbol{\kappa} \cdot \boldsymbol{\nu}+\lambda \mathbf{g}) d s \mid\right.$

$$
\left.\boldsymbol{\kappa} \in C\left(\Gamma_{1}, \mathbf{E}_{s}^{n}\right)\right\}-\int_{\Gamma_{1}} \lambda \mathbf{g} \cdot \boldsymbol{\mu}+c_{\varepsilon(\mathbf{u})_{\mid \bar{\Omega}}}
$$

$=\int_{\Gamma_{1}} j_{\infty}\left(x, \frac{d\left(\left(\boldsymbol{\mu}-\gamma_{B}^{I}(\mathbf{u}) d s\right) \otimes_{s} \boldsymbol{\nu}\right)}{d\left|\left(\boldsymbol{\mu}-\gamma_{B}^{I}(\mathbf{u}) d s\right) \otimes_{s} \boldsymbol{\nu}\right|}\right) d\left|\left(\boldsymbol{\mu}-\gamma_{B}^{I}(\mathbf{u}) d s\right) \otimes_{s} \boldsymbol{\nu}\right|$

$-\int_{\Gamma_{1}} \lambda \mathbf{g} \cdot \boldsymbol{\mu}+c_{\varepsilon(\mathbf{u})_{\mid \bar{\Omega}}}$

for all $\boldsymbol{\mu} \in \mathbb{M}_{b}\left(\Gamma_{1}, \mathbb{R}^{n}\right)$. Thus we get (2.47). Since $\left(\widetilde{\mathbb{H}}_{\lambda}^{j}\right)^{* *}\left(\varepsilon(\mathbf{u})_{\mid \bar{\Omega}}, \boldsymbol{\mu} \otimes_{s} \boldsymbol{\nu}\right)$ $=\left(\mathbb{H}_{\lambda}^{j}\right)^{* *}\left(\varepsilon(\mathbf{u})_{\mid \bar{\Omega}}, \boldsymbol{\mu} \otimes_{s} \boldsymbol{\nu}\right)$ for every $\left(\varepsilon(\mathbf{u})_{\mid \bar{\Omega}}, \boldsymbol{\mu}\right) \in \mathbf{Y}^{1}(\bar{\Omega}) \times L^{1}\left(\Gamma_{1}, \mathbb{R}^{n}\right)$, and $\left(\mathbb{H}_{\lambda}^{j}\right)^{* *}$ is the l.s.c. regularization of $\mathbb{H}_{\lambda}^{j}$, we obtain (2.43).

Now we turn to mechanical conclusions. 
The following is known as the Suquet problem (see [12]):

$$
\begin{aligned}
\left(S Q_{\lambda, j}\right) \text { Find } \inf \left\{\lambda F_{S Q}(\mathbf{u}, \boldsymbol{\mu})\right. & +G_{j}(\varepsilon(\mathbf{u})) \mid \\
& \left.\mathbf{u} \in L D(\Omega), \boldsymbol{\mu} \in \mathbb{M}_{b}\left(\Gamma_{1}, \mathbb{R}^{n}\right)\right\},
\end{aligned}
$$

where the functional of the total loading has the form

$$
\begin{aligned}
\lambda F_{S Q}(\mathbf{u}, \boldsymbol{\mu}) \equiv & -\lambda\left(\int_{\Omega} \mathbf{f} \cdot \mathbf{u} d x+\int_{\Gamma_{1}} \mathbf{g} \cdot \boldsymbol{\mu}\right) \\
& +\int_{\Gamma_{0}} I_{\left\{\gamma_{B}(\mathbf{u})(x)=\mathbf{0}\right\}}\left(-\gamma_{B}(\mathbf{u}) \otimes_{s} \boldsymbol{\nu}\right) d s
\end{aligned}
$$

if $\boldsymbol{\mu}=\boldsymbol{\gamma}_{B}^{I}(\mathbf{u}) d s$ for $\left|\boldsymbol{\mu}-\gamma_{B}^{I}(\mathbf{u}) d s\right|$-a.e. $x \in \Gamma_{1}$, and $\lambda F_{S Q}(\mathbf{u}, \boldsymbol{\mu}) \equiv \infty$ otherwise. The elastic-plastic potential $G_{j}$ is defined by $[2,(3.11)]$.

The following bidual relaxed Suquet problem is studied in [1]:

$$
\begin{array}{r}
\left(S P_{\lambda, j}^{* *}\right) \text { Find } \inf \left\{\left(\lambda F_{H}\right)^{* *}(\mathbf{u}, \boldsymbol{\mu})+G_{j}^{* *}(\varepsilon(\mathbf{u})) \mid\right. \\
\left.\mathbf{u} \in B D(\Omega), \boldsymbol{\mu} \in \mathbb{M}_{b}\left(\Gamma_{1}, \mathbb{R}^{n}\right)\right\},
\end{array}
$$

where for every $\mathbf{u} \in B D(\Omega)$ and $\boldsymbol{\mu} \in \mathbb{M}_{b}\left(\Gamma_{1}, \mathbb{R}^{n}\right)$,

$$
\begin{aligned}
\left(\lambda F_{H}\right)^{* *}(\mathbf{u}, \boldsymbol{\mu}) \equiv & -\lambda\left[\int_{\Omega} \mathbf{f} \cdot \mathbf{u} d x+\int_{\Gamma_{1}} \mathbf{g} \cdot \boldsymbol{\mu}\right] \\
& +\int_{\Gamma_{0}} j_{\infty}\left(x,\left(-\gamma_{B}(\mathbf{u}) \otimes_{s} \boldsymbol{\nu}\right)\right) d s \\
+ & \int_{\Gamma_{1}} j_{\infty}\left(x, \frac{d\left(\left(\boldsymbol{\mu}-\gamma_{B}(\mathbf{u}) d s\right) \otimes_{s} \boldsymbol{\nu}\right)}{d\left|\left(\boldsymbol{\mu}-\gamma_{B}(\mathbf{u}) d s\right) \otimes_{s} \boldsymbol{\nu}\right|}\right) d\left|\left(\boldsymbol{\mu}-\gamma_{B}(\mathbf{u}) d s\right) \otimes_{s} \boldsymbol{\nu}\right|
\end{aligned}
$$

and $G_{j}^{* *}(\varepsilon(\cdot))$ is defined in $[2,(5.4)]$.

Assumption 7. There exist $k_{b}>0$ and $r_{1}>0$ such that $j^{*}\left(x, \mathbf{w}^{*}\right) \leq k_{b}$ for every $\mathbf{w}^{*} \in B_{\mathbf{E}_{s}^{n}}\left(0, r_{1}\right)$ and for $d x$-a.e. $x \in \Omega$, where $B_{\mathbf{E}_{s}^{n}}\left(0, r_{1}\right)$ is the closed ball in $\mathbf{E}_{s}^{n}$ with center 0 and radius $r_{1}$.

Let the functional

$$
(\mathbf{u}, \boldsymbol{\mu}) \mapsto\left\lceil S Q_{\lambda, j}\right\rceil(\mathbf{u}, \boldsymbol{\mu}) \equiv \lambda F_{S Q}(\mathbf{u}, \boldsymbol{\mu})+G_{j}(\varepsilon(\mathbf{u}))
$$

be coercive over $B D(\Omega) \times \mathbb{M}_{b}\left(\Gamma_{1}, \mathbb{R}^{n}\right)$, i.e.,

$$
\text { if }\left\|\mathbf{u}_{m}\right\|_{B D}+\left\|\boldsymbol{\mu}_{m}\right\|_{\mathbb{M}_{b}} \rightarrow \infty \text { then }\left\lceil S Q_{\lambda, j}\right\rceil\left(\mathbf{u}_{m}, \boldsymbol{\mu}_{m}\right) \rightarrow \infty
$$

for any sequences $\left\{\mathbf{u}_{m}\right\}_{m \in \mathbb{N}} \subset B D(\Omega)$ and $\left\{\boldsymbol{\mu}_{m}\right\}_{m \in \mathbb{N}} \subset \mathbb{M}_{b}\left(\Gamma_{1}, \mathbb{R}^{n}\right)$. Moreover, let $0 \leq \lambda_{1}<\lambda$. Then the functional $\left\lceil S Q_{\lambda_{1}, j}\right\rceil$ is coercive on $B D(\Omega) \times$ $\mathbb{M}_{b}\left(\Gamma_{1}, \mathbb{R}^{n}\right)$. Similarly, if the functional

$$
(\mathbf{u}, \boldsymbol{\mu}) \mapsto\left\lceil S P_{\lambda, j}^{* *}\right\rceil(\mathbf{u}, \boldsymbol{\mu}) \equiv\left(\lambda F_{H}\right)^{* *}(\mathbf{u}, \boldsymbol{\mu})+G_{j}^{* *}(\varepsilon(\mathbf{u}))
$$

is coercive and $0 \leq \lambda_{1} \leq \lambda$, then $\left\lceil S P_{\lambda_{1}, j}^{* *}\right\rceil$ is coercive over $B D(\Omega) \times$ $\mathbb{M}_{b}\left(\Gamma_{1}, \mathbb{R}^{n}\right)$. Moreover, we obtain $\left\lceil S Q_{\lambda, j}\right\rceil \geq\left\lceil S P_{\lambda, j}^{* *}\right\rceil$. 
We say that a net $\left\{\left(\mathbf{u}_{t}, \boldsymbol{\mu}_{t}\right)\right\}_{t \in T} \subset B D(\Omega) \times \mathbb{M}_{b}\left(\Gamma_{1}, \mathbb{R}^{n}\right)$ converges to $\left(\mathbf{u}_{0}, \boldsymbol{\mu}_{0}\right)$ in the weak* $B D(\Omega) \times \mathbb{M}_{b}\left(\Gamma_{1}, \mathbb{R}^{n}\right)$ topology if $\mathbf{u}_{t} \rightarrow \mathbf{u}_{0}$ in the weak* $B D(\Omega)$ topology and $\boldsymbol{\mu}_{t} \rightarrow \boldsymbol{\mu}_{0}$ in the weak* $\mathbb{M}_{b}\left(\Gamma_{1}, \mathbb{R}^{n}\right)$ topology.

Theorem 8. Let $d s\left(\Gamma_{0}\right) \neq 0$ and $\mathbf{f} \in L^{n+\delta}\left(\Omega, \mathbb{R}^{n}\right)$, where $\delta \geq 0$. If the functional $\left\lceil S Q_{\lambda, j}\right\rceil$ is coercive over $B D(\Omega) \times \mathbb{M}_{b}\left(\Gamma_{1}, \mathbb{R}^{n}\right)$, then the l.s.c. regularization of $\left\lceil S Q_{\lambda, j}\right\rceil$ in the weak $B D(\Omega) \times \mathbb{M}_{b}\left(\Gamma_{1}, \mathbb{R}^{n}\right)$ topology is the functional $\left\lceil S P_{\lambda, j}^{* *}\right\rceil$ (cf. (2.50), (2.52), (2.53)).

Proof. Step 1. Let $(\widetilde{\mathbf{u}}, \widetilde{\boldsymbol{\mu}}) \in B D(\Omega) \times \mathbb{M}_{b}\left(\Gamma_{1}, \mathbb{R}^{n}\right)$ and $\left\{\left(\mathbf{u}_{p}, \boldsymbol{\mu}_{p}\right)\right\}_{p \in P}$ be a net such that $\left(\mathbf{u}_{p}, \boldsymbol{\mu}_{p}\right) \rightarrow(\widetilde{\mathbf{u}}, \widetilde{\boldsymbol{\mu}})$ in the weak ${ }^{*} B D(\Omega) \times \mathbb{M}_{b}\left(\Gamma_{1}, \mathbb{R}^{n}\right)$ topology. By Steps 1 and 2 of [2, proof of Theorem 18], $\liminf _{p \in P}\left\lceil S Q_{\lambda, j}\right\rceil\left(\mathbf{u}_{p}, \boldsymbol{\mu}_{p}\right) \geq$ $\left\lceil S P_{\lambda, j}^{* *}\right\rceil(\widetilde{\mathbf{u}}, \widetilde{\boldsymbol{\mu}})$, since $\left\lceil S Q_{\lambda, j}\right\rceil$ is equal to the function defined in $[2,(3.8)]$ and $\left\lceil R P_{\lambda, j}^{* *}\right\rceil$ is equal to $\left\lceil S P_{\lambda, j}^{* *}\right\rceil$ (cf. $[2,(5.6)]$ ) if $\boldsymbol{\mu}=\gamma_{B}(\mathbf{u}) d s$ on $\Gamma_{1}$.

Step 2. Let $\left(\widetilde{\mathbb{H}}_{\lambda}^{j}\right)^{* *}\left(\varepsilon(\widetilde{\mathbf{u}})_{\mid \bar{\Omega}}, \widetilde{\boldsymbol{\mu}} \otimes_{s} \boldsymbol{\nu}\right)<\infty$. By Theorem 7 , there is a net $\left\{\left(\mathbf{u}_{t}, \boldsymbol{\mu}_{t}\right)\right\}_{t \in \Sigma} \subset B D(\Omega) \times \mathbb{M}_{b}\left(\Gamma_{1}, \mathbb{R}^{n}\right)$ such that $\left(\mathbf{u}_{t}, \boldsymbol{\mu}_{t} \otimes_{s} \boldsymbol{\nu}\right) \rightarrow\left(\widetilde{\mathbf{u}}, \widetilde{\boldsymbol{\mu}} \otimes_{s} \boldsymbol{\nu}\right)$ in the topology $\sigma\left(\mathbf{Y}^{1}(\bar{\Omega}) \times \mathbf{M}^{1}\left(\Gamma_{1}\right), C_{\operatorname{div}}\left(\bar{\Omega}, \mathbf{E}_{s}^{n}\right) \times C\left(\Gamma_{1}, \mathbf{E}_{s}^{n}\right)\right)$, where the bilinear form $\langle\cdot ; \cdot\rangle_{2}$ between $\mathbf{Y}^{1}(\bar{\Omega}) \times \mathbf{M}^{1}\left(\Gamma_{1}\right)$ and $C_{\mathrm{div}}\left(\bar{\Omega}, \mathbf{E}_{s}^{n}\right) \times C\left(\Gamma_{1}, \mathbf{E}_{s}^{n}\right)$ is given by (2.8). Moreover,

$$
\lim _{t \in \Sigma}\left(\left\lceil S Q_{\lambda, j}\right\rceil\left(\mathbf{u}_{t}, \boldsymbol{\mu}_{t}\right)+\lambda \int_{\Omega} \mathbf{f} \cdot \mathbf{u}_{t} d x\right)=\left(\widetilde{\mathbb{H}}_{\lambda}^{j}\right)^{* *}\left(\varepsilon(\widetilde{\mathbf{u}})_{\mid \bar{\Omega}}, \widetilde{\boldsymbol{\mu}} \otimes_{s} \boldsymbol{\nu}\right) .
$$

Theorem 7 holds in the special case when $\mathbf{g}=\mathbf{0}$ on $\Gamma_{1}$. Then

$$
\begin{aligned}
\lim _{t \in \Sigma}\left[\left\lceil S Q_{\lambda, j}\right\rceil\left(\mathbf{u}_{t}, \boldsymbol{\mu}_{t}\right)\right. & \left.+\lambda\left(\int_{\Omega} \mathbf{f} \cdot \mathbf{u}_{t} d x+\int_{\Gamma_{1}} \mathbf{g} \cdot \boldsymbol{\mu}_{t}\right)\right] \\
= & \left(\widetilde{\mathbb{H}}_{\lambda}^{j}\right)^{* *}\left(\varepsilon(\widetilde{\mathbf{u}})_{\mid \bar{\Omega}}, \widetilde{\boldsymbol{\mu}} \otimes_{s} \boldsymbol{\nu}\right)+\lambda \int_{\Gamma_{1}} \mathbf{g} \cdot \widetilde{\boldsymbol{\mu}} \in \mathbb{R},
\end{aligned}
$$

because $\left|\lambda \int_{\Gamma_{1}} \mathbf{g} \cdot \tilde{\boldsymbol{\mu}}\right|<\infty$. By Assumption 7, [2, Lemma 16] and (2.58), the net $\left\{\left(\mathbf{u}_{t}, \boldsymbol{\mu}_{t}\right)\right\}_{t \in \Sigma}$ is bounded and $\left(\mathbf{u}_{t}, \boldsymbol{\mu}_{t}\right) \rightarrow(\widetilde{\mathbf{u}}, \widetilde{\boldsymbol{\mu}})$ in the weak* $B D(\Omega) \times$ $\mathbb{M}_{b}\left(\Gamma_{1}, \mathbb{R}^{n}\right)$ topology. Therefore, by Theorem 7 and [2, Lemma 15], we get

$$
\lim _{t \in \Sigma}\left\lceil S Q_{\lambda, j}\right\rceil\left(\mathbf{u}_{t}, \boldsymbol{\mu}_{t}\right)=\left\lceil S P_{\lambda, j}^{* *}\right\rceil(\widetilde{\mathbf{u}}, \widetilde{\boldsymbol{\mu}}) .
$$

Step 3. Let $\left(\widetilde{\mathbb{H}}_{\lambda}^{j}\right)^{* *}\left(\varepsilon(\widetilde{\mathbf{u}})_{\mid \bar{\Omega}}, \widetilde{\boldsymbol{\mu}} \otimes_{s} \boldsymbol{\nu}\right)=\infty$. Then, by Step 4 of the proof of Theorem 18 from [2], for every net $\left\{\left(\mathbf{u}_{p}, \boldsymbol{\mu}_{p}\right)\right\}_{p \in P}$ such that $\left(\mathbf{u}_{p}, \boldsymbol{\mu}_{p}\right) \rightarrow$ $(\widetilde{\mathbf{u}}, \widetilde{\boldsymbol{\mu}})$ in the weak ${ }^{*} B D(\Omega) \times \mathbb{M}_{b}\left(\Gamma_{1}, \mathbb{R}^{n}\right)$ topology, we have

$$
\liminf _{p \in P}\left\lceil S Q_{\lambda, j}\right\rceil\left(\mathbf{u}_{t}, \boldsymbol{\mu}_{t}\right)=\infty=\left\lceil S P_{\lambda, j}^{* *}\right\rceil(\widetilde{\mathbf{u}}, \widetilde{\boldsymbol{\mu}}) .
$$

Next, we proceed similarly to Step 5 of the proof of [2, Theorem 18].

Corollary 9. $\left\lceil S Q_{\lambda, j}\right\rceil$ is coercive over $B D(\Omega) \times \mathbb{M}_{b}\left(\Gamma_{1}, \mathbb{R}^{n}\right)$ if and only if $\left\lceil S P_{\lambda, j}^{* *}\right\rceil$ is coercive.

Proof. We prove this similarly to [2, Corollary 19]. 
COROLlary 10. The functional (3.8) from [2] is coercive over $B D(\Omega)$ if and only if $\left\lceil S P_{\lambda, j}^{* *}\right\rceil$ is coercive, where $\mathbf{u}^{0}=\mathbf{0}$ on $\Gamma_{0}$ (in the functional $[2$, $(3.8)])$.

Proof. Indeed, by $[2,(3.11)],(2.51)$ and $(2.54)$, the functional $\left\lceil S Q_{\lambda, j}\right\rceil$ is coercive over $B D(\Omega) \times \mathbb{M}_{b}\left(\Gamma_{1}, \mathbb{R}^{n}\right)$ if and only if (3.8) from [2] is coercive over $B D(\Omega)$.

Theorem 11. Let $d s\left(\Gamma_{0}\right) \neq 0$ and $\mathbf{f} \in L^{n+\delta}\left(\Omega, \mathbb{R}^{n}\right)$, where $\delta \geq 0$. Moreover, let $j^{*}: \Omega \times \mathbf{E}_{s}^{n} \rightarrow \mathbb{R} \cup\{\infty\}$ be a nonnegative normal integrand. If the functional (3.8) from [2] is coercive over $B D(\Omega)$ and $\mathbf{u}^{0}=\mathbf{0}$ on $\Gamma_{0}$ (in $[2$, (3.8)]), then the couple $(\widetilde{\mathbf{u}}, \widetilde{\boldsymbol{\mu}}) \in B D(\Omega) \times \mathbb{M}_{b}\left(\Gamma_{1}, \mathbb{R}^{n}\right)$ is a solution of the problem $\left(S P_{\lambda, j}^{* *}\right)$ if and only if $\widetilde{\boldsymbol{\mu}}=\gamma_{B}(\widetilde{\mathbf{u}})_{\mid \Gamma_{1}}$ ds $\in \mathbb{M}_{b}\left(\Gamma_{1}, \mathbb{R}^{n}\right)$ and $\widetilde{\mathbf{u}}$ is a solution of the problem $\left(R P_{\lambda, j}^{* *}\right)$ (cf. $[2,(5.2)-(5.4)],(2.52)$ and $\left.(2.53)\right)$.

Proof. Since the normal integrand $j^{*}$ is nonnegative, $\left(R P_{\lambda, j}^{* *}\right)$ and $\left(S P_{\lambda, j}^{* *}\right)$ have finite infima, because $\left\lceil R P_{\lambda, j}^{* *}\right\rceil(\mathbf{0})<\infty$ and $\left\lceil S P_{\lambda, j}^{* *}\right\rceil(\mathbf{0}, \mathbf{0})<\infty$ (cf. $[2$, (5.6)]).

First, let $\widetilde{\mathbf{u}}$ be a solution of $\left(R P_{\lambda, j}^{* *}\right)$. Then the couple $\left(\widetilde{\mathbf{u}}, \gamma_{B}(\widetilde{\mathbf{u}})_{\mid \Gamma_{1}} d s\right) \in$ $B D(\Omega) \times \mathbb{M}_{b}\left(\Gamma_{1}, \mathbb{R}^{n}\right)$ is a solution of $\left(S P_{\lambda, j}^{* *}\right)$, since

$$
\inf \left(R P_{\lambda, j}^{* *}\right)=\inf \left(P_{\lambda, j}\right)=\inf \left(S Q_{\lambda, j}\right)=\inf \left(S P_{\lambda, j}^{* *}\right),
$$

where $\left(P_{\lambda, j}\right)$ is defined in $[2,(5.1)$ and (3.8)] (cf. [2, Theorem 18], Theorem 8, $[2,(3.8),(5.2),(5.1)]$ and $(2.50),(2.52))$.

Next, we show the inverse implication. Let $(\widetilde{\mathbf{u}}, \widetilde{\boldsymbol{\mu}})$ be a solution of $\left(S P_{\lambda, j}^{* *}\right)$. Then there exists a sequence $\left\{\left(\mathbf{u}_{m}, \boldsymbol{\mu}_{m}\right)\right\}_{m \in \mathbb{N}}$ in $B D(\Omega) \times \mathbb{M}_{b}\left(\Gamma_{1}, \mathbb{R}^{n}\right)$ such that $\lim _{m \rightarrow \infty}\left\lceil S Q_{\lambda, j}\right\rceil\left(\mathbf{u}_{m}, \boldsymbol{\mu}_{m}\right)=\inf \left(S P_{\lambda, j}^{* *}\right)$ and $\left(\mathbf{u}_{m}, \boldsymbol{\mu}_{m}\right) \rightarrow(\widetilde{\mathbf{u}}, \widetilde{\boldsymbol{\mu}})$ in weak $^{*} B D(\Omega) \times \mathbb{M}_{b}\left(\Gamma_{1}, \mathbb{R}^{n}\right)$ topology. By (2.50), there exists $m_{0} \in \mathbb{N}$ such that for every $m>m_{0}$ we have $\boldsymbol{\mu}_{m}=\gamma_{B}\left(\mathbf{u}_{m}\right) d s$ on $\Gamma_{1}$, because inf $\left(S P_{\lambda, j}^{* *}\right)$ $<\infty$. Since $\inf \left(R P_{\lambda, j}^{* *}\right)=\inf \left(S P_{\lambda, j}^{* *}\right)$, we obtain

$$
\lim _{m \rightarrow \infty}\left\lceil S Q_{\lambda, j}\right\rceil\left(\mathbf{u}_{m}, \boldsymbol{\gamma}_{B}\left(\mathbf{u}_{m}\right)_{\mid \Gamma_{1}} d s\right)=\lim _{m \rightarrow \infty} P_{\lambda, j}\left(\mathbf{u}_{m}\right)=\inf \left\lceil R P_{\lambda, j}^{* *}\right\rceil,
$$

where $P_{\lambda, j}$ is defined in [2, (3.8)] (cf. (2.61)). Therefore, by [2, Theorem 18], we get

$$
\left\lceil R P_{\lambda, j}^{* *}\right\rceil(\widetilde{\mathbf{u}})=\inf \left(R P_{\lambda, j}^{* *}\right)=\inf \left(S P_{\lambda, j}^{* *}\right) .
$$

Reasoning by contradiction, we prove that $\widetilde{\boldsymbol{\mu}}=\gamma_{B}(\widetilde{\mathbf{u}}) d s$ on $\Gamma_{1}$. Suppose $\widetilde{\boldsymbol{\mu}} \neq$ $\gamma_{B}(\widetilde{\mathbf{u}})_{\mid \Gamma_{1}} d s$. By $[2,(5.2)],(2.52)$ and $(2.63)$ we have $\left\lceil S P_{\lambda, j}^{* *}\right\rceil\left(\widetilde{\mathbf{u}}, \gamma_{B}(\widetilde{\mathbf{u}})_{\mid \Gamma_{1}} d s\right)$ $=\left\lceil S P_{\lambda, j}^{* *}\right\rceil(\widetilde{\mathbf{u}}, \widetilde{\boldsymbol{\mu}})$. The function

$$
\begin{aligned}
{[0, \infty) \ni t \mapsto } & \int_{\Gamma_{1}} j_{\infty}\left(x, \frac{d\left(t\left(\widetilde{\boldsymbol{\mu}}-\gamma_{B}(\widetilde{\mathbf{u}}) d s\right) \otimes_{s} \boldsymbol{\nu}\right)}{d\left|\left(\widetilde{\boldsymbol{\mu}}-\gamma_{B}(\widetilde{\mathbf{u}}) d s\right) \otimes_{s} \boldsymbol{\nu}\right|}\right) d\left|\left(\widetilde{\boldsymbol{\mu}}-\gamma_{B}(\widetilde{\mathbf{u}}) d s\right) \otimes_{s} \boldsymbol{\nu}\right| \\
& +(1-t) \int_{\Gamma_{1}} \mathbf{g} \cdot \boldsymbol{\gamma}_{B}(\widetilde{\mathbf{u}}) d s+t \int_{\Gamma_{1}} \mathbf{g} \cdot \widetilde{\boldsymbol{\mu}}
\end{aligned}
$$


is positively homogeneous. Then for every $t>0,\left\lceil S P_{\lambda, j}^{* *}\right\rceil(\widetilde{\mathbf{u}}, \widetilde{\boldsymbol{\mu}})=\left\lceil S P_{\lambda, j}^{* *}\right\rceil(\widetilde{\mathbf{u}}$, $\left.t\left(\widetilde{\boldsymbol{\mu}}-\boldsymbol{\gamma}_{B}(\widetilde{\mathbf{u}})_{\mid \Gamma_{1}} d s\right)+\boldsymbol{\gamma}_{B}(\widetilde{\mathbf{u}})_{\mid \Gamma_{1}} d s\right)$. Therefore we obtain a contradiction with the coercivity of $\left\lceil S P_{\lambda, j}^{* *}\right\rceil$ (cf. Corollary 10).

Let $d s\left(\Gamma_{0}\right)=0$ and $L(\widehat{\mathbf{u}})=0$ for every $\widehat{\mathbf{u}} \in \mathcal{R}_{0} \equiv\{\mathbf{u} \in B D \mid \varepsilon(\mathbf{u})=\mathbf{0}\}$. Moreover, let the functionals (3.8) from [2] and $\left(R P_{\lambda, j}^{* *}\right)$ be defined over $B D(\Omega) / \mathcal{R}_{0}$, and let $\left(S Q_{\lambda, j}\right)$ and $\left(S P_{\lambda, j}^{* *}\right)$ be defined over the space $(B D(\Omega) \times$ $\left.\mathbb{M}_{b}\left(\Gamma_{1}, \mathbb{R}^{n}\right)\right) /\left\{\left(\widehat{\mathbf{u}}, \gamma_{B}(\widehat{\mathbf{u}})_{\mid \Gamma_{1}} d s\right) \mid \widehat{\mathbf{u}} \in \mathcal{R}_{0}\right\}$. Then the assertions of Theorem 8 , Corollary 9, Corollary 10 and Theorem 11 hold. Indeed, in their proofs, we can replace weak ${ }^{*} B D(\Omega)$ topology by $\sigma\left(\mathbf{Y}^{1}(\bar{\Omega}), C_{\operatorname{div}}\left(\bar{\Omega}, \mathbf{E}_{s}^{n}\right)\right)$, and $G_{j}(\varepsilon(\cdot))$ does not depend on $\mathbf{u} \in \operatorname{ker} \boldsymbol{\varepsilon}$ (cf. [2, Lemma 15], (2.10) and (2.14)).

3. The limit analysis problem. Here we define the limit analysis problem $\left(S P_{0, j}^{* *}\right)_{A L}$ associated with $\left(S P_{\lambda, j}^{* *}\right)$. We prove that $\left(S P_{\lambda, j}^{* *}\right)$ is coercive if inf $\left(S P_{0, j}^{* *}\right)_{A L}>\lambda$, under some assumptions given below. Moreover, we obtain an existence theorem for $\left(S P_{0, j}^{* *}\right)_{A L}$.

Assumption 8. $j^{*}$ is a nonnegative function.

The original problem $\left(P_{\lambda, j}\right)$ is defined by (see [2, formula (3.8)-(3.11)])

$$
\left(P_{\lambda, j}\right) \text { find } \inf \left\{\lambda F(\mathbf{u})+G_{j}(\varepsilon(\mathbf{u})) \mid \mathbf{u} \in B D(\Omega)\right\},
$$

where

$$
\lambda F(\mathbf{u}) \equiv-\lambda L(\mathbf{u})+I_{C_{a}(\mathbf{0})}(\mathbf{u}), \quad L(\mathbf{u}) \equiv \int_{\Omega} \mathbf{f} \cdot \mathbf{u} d x+\int_{\Gamma_{1}} \mathbf{g} \cdot \gamma_{B}(\mathbf{u}) d s
$$

and

$$
C_{a}(\mathbf{0}) \equiv\left\{\mathbf{u} \in B D(\Omega) \mid \boldsymbol{\gamma}_{B}(\mathbf{u})_{\mid \Gamma_{0}}=\mathbf{0} \text { on } \Gamma_{0}\right\} .
$$

The elastic-plastic potential $G_{j}: \mathbb{M}_{b}\left(\Omega, \mathbf{E}_{s}^{n}\right) \rightarrow \mathbb{R} \cup\{\infty\}$ is defined by

$$
G_{j}(\boldsymbol{\mu}) \equiv \begin{cases}\int_{\Omega} j(x, \boldsymbol{\mu}) d x & \text { if } \boldsymbol{\mu} \in L^{1}\left(\Omega, \mathbf{E}_{s}^{n}\right) \\ \infty & \text { otherwise }\end{cases}
$$

With $\left(P_{\lambda, j}\right)$ one associates the limit analysis problem $\left(P_{0, j}\right)_{A L}$ :

$$
\begin{aligned}
\left(P_{0, j}\right)_{A L} \quad \text { find } \inf \left\{\int_{\Omega} j_{\infty}(x, \varepsilon(\mathbf{u})) d x \mid \mathbf{u}\right. & \in L D(\Omega), \\
\gamma_{B}(\mathbf{u}) & \left.=\mathbf{0} \text { on } \Gamma_{0}, L(\mathbf{u})=1\right\} .
\end{aligned}
$$

Similarly, $\left(S P_{\lambda, j}^{* *}\right)$, defined in (2.52), is connected with the relaxed limit analysis problem $\left(S P_{0, j}^{* *}\right)_{A L}$ :

$$
\begin{aligned}
& \left(S P_{0, j}^{* *}\right)_{A L} \quad \text { find inf }\left\{\int_{\Gamma_{0}} j_{\infty}\left(x,-\gamma_{B}(\mathbf{u}) \otimes_{s} \boldsymbol{\nu}\right) d s\right. \\
& \quad+\int_{\Gamma_{1}} j_{\infty}\left(x, \frac{d\left(\left(\boldsymbol{\mu}-\gamma_{B}(\mathbf{u}) d s\right) \otimes_{s} \boldsymbol{\nu}\right)}{d\left|\left(\boldsymbol{\mu}-\gamma_{B}(\mathbf{u}) d s\right) \otimes_{s} \boldsymbol{\nu}\right|}\right) d\left|\left(\boldsymbol{\mu}-\gamma_{B}(\mathbf{u}) d s\right) \otimes_{s} \boldsymbol{\nu}\right|
\end{aligned}
$$




$$
\begin{aligned}
+\int_{\Omega} j_{\infty}\left(x, \boldsymbol{\varepsilon}(\mathbf{u})_{a}\right) d x+\int_{\Omega} j_{\infty}\left(x, \frac{d \varepsilon(\mathbf{u})_{s}}{d\left|\boldsymbol{\varepsilon}(\mathbf{u})_{s}\right|}\right) d\left|\varepsilon(\mathbf{u})_{s}\right| & \\
\mathbf{u} & \left.\in B D(\Omega), \boldsymbol{\mu} \in \mathbb{M}_{b}\left(\Gamma_{1}, \mathbb{R}^{n}\right), \int_{\Omega} \mathbf{f} \cdot \mathbf{u} d x+\int_{\Gamma_{1}} \mathbf{g} \cdot \boldsymbol{\mu}=1\right\} .
\end{aligned}
$$

Directly from (3.5) and (3.6) we get

$$
\inf \left(P_{0, j}\right)_{A L} \geq \inf \left(S P_{0, j}^{* *}\right)_{A L} .
$$

If $d s\left(\Gamma_{0}\right)=0$ and $\inf \left(P_{0, j}\right)_{A L}>0$ then for every $\mathbf{u} \in L D(\Omega)$ and $\overline{\mathbf{u}} \in$ $L D(\Omega)$ such that $\varepsilon(\overline{\mathbf{u}})=\mathbf{0}$ in $\Omega$, we have $L(\mathbf{u})=L(\mathbf{u}+\overline{\mathbf{u}})$, since $j^{*}$ is nonnegative (i.e. $j(x, \mathbf{0}) \leq 0$ for $d x$-a.e. $x \in \Omega$ ); cf. Assumption 8 and (3.2)). We can prove the following result.

Proposition 12. Assume that $\bar{\lambda}_{r}$ satisfies Assumption 5 , where $\lambda$ is replaced by $\bar{\lambda}_{r}$. Moreover, let $\mathbf{u}^{0}=\mathbf{0}$ in $\left(P_{\lambda_{r}, j}\right)$. If $\inf \left(P_{0, j}\right)_{A L}>\bar{\lambda}_{r}>\lambda_{r} \geq 0$ then $\inf \left(P_{\lambda_{r}, j}\right)>-\infty$. The inverse implication holds in the following form: if $\inf \left(P_{\lambda_{r}, j}\right)>-\infty$ then $\inf \left(P_{0, j}\right)_{A L} \geq \lambda_{r}$. Moreover, if $\inf \left(P_{0, j}\right)_{A L}>$ $\bar{\lambda}_{r}>\lambda_{r} \geq 0$ then any sequence $\left\{\mathbf{u}_{m}\right\}_{m \in \mathbb{N}} \subset B D(\Omega)$ such that

$$
\inf _{\mathbf{z}}\left\{\left\|\mathbf{u}_{m}+\mathbf{z}\right\|_{B D} \mid \mathbf{z} \in B D(\Omega) \text { and } \boldsymbol{\varepsilon}(\mathbf{z})=\mathbf{0}\right\} \rightarrow \infty
$$

satisfies $\lim _{m \rightarrow \infty}\left(\lambda_{r} F\left(\mathbf{u}_{m}\right)+G_{j}\left(\varepsilon\left(\mathbf{u}_{m}\right)\right)\right)=\infty$, i.e. the functional $B D(\Omega) \ni$ $\mathbf{u} \mapsto\left(\lambda_{r} F(\mathbf{u})+G_{j}(\varepsilon(\mathbf{u}))\right)$ is coercive.

Below we study the relation between $\left(S P_{\lambda, j}^{* *}\right)$ and the relaxed limit analysis problem $\left(S P_{0, j}^{* *}\right)_{A L}$.

REMARK 1. If $\inf \left(P_{0, j}\right)_{A L}>0$, then the assumptions of Corollaries 9, 10 and Theorem 8 hold (in the sense of $(3.8))$ if $d s\left(\Gamma_{0}\right)=0$. Indeed, $L(\widehat{\mathbf{u}})=0$ for $\varepsilon(\widehat{\mathbf{u}})=\mathbf{0}$ (cf. Assumption 8 and (3.2)).

Proposition 13. Assume that $\bar{\lambda}_{r}$ satisfies Assumption 5, where $\lambda$ is replaced by $\bar{\lambda}_{r}$, and suppose $\inf \left(P_{0, j}\right)_{A L}>\bar{\lambda}_{r}>\lambda_{r} \geq 0$. Problem $\left(S P_{\lambda_{r}, j}^{* *}\right)$ is coercive if and only if $\inf \left(S P_{0, j}^{* *}\right)_{A L}>\lambda_{r}$.

Proof. By Corollary 10, problem $\left(S P_{\lambda, j}^{* *}\right)$ is coercive if and only if $\left(P_{\lambda, j}\right)$ is coercive. By Proposition 12, if $\bar{\lambda}_{r}$ satisfies Assumption 5 and $\inf \left(P_{0, j}\right)_{A L}>$ $\bar{\lambda}_{r}>\lambda_{r} \geq 0$, then $\left(P_{\lambda_{r}, j}\right)$ is coercive if and only if $\left(P_{\lambda_{r}, j_{\infty}}\right)$ is coercive. From Corollary 10, $\left(P_{\lambda, j_{\infty}}\right)$ is coercive if and only if $\left(S P_{\lambda, j_{\infty}}^{* *}\right)$ is coercive. Therefore, if $\bar{\lambda}_{r}$ satisfies Assumption 5 and $\inf \left(P_{0, j}\right)_{A L}>\bar{\lambda}_{r}>\lambda_{r} \geq 0$, then $\left(S P_{\lambda_{r}, j}^{* *}\right)$ is coercive if and only if $\left(S P_{\lambda_{r}, j_{\infty}}^{* *}\right)$ is coercive. Moreover, $\left(S P_{\lambda, j_{\infty}}^{* *}\right)$ is coercive if and only if $\left(S P_{0, j}^{* *}\right)_{A L}>\lambda$, where $\lambda \geq 0$.

TheOREM 14. There exists a solution $(\widehat{\mathbf{u}}, \widehat{\boldsymbol{\mu}}) \in B D(\Omega) \times \mathbb{M}_{b}\left(\Gamma_{1}, \mathbb{R}^{n}\right)$ of the problem $\left(S P_{0, j}^{* *}\right)_{A L}$, i.e. $\left(S P_{0, j}^{* *}\right)_{A L}$ achieves its infimum at the point $(\widehat{\mathbf{u}}, \widehat{\boldsymbol{\mu}})$. If $d s\left(\Gamma_{0}\right)=0$ then we assume that $L(\widehat{\mathbf{u}})=0$ for every $\widehat{\mathbf{u}} \in \mathcal{R}_{0} \equiv$ $\{\mathbf{u} \in B D \mid \varepsilon(\mathbf{u})=\mathbf{0}\}$. 
Proof. The functional $\left(S P_{\lambda, j_{\infty}}^{* *}\right)$ is l.s.c. in the weak ${ }^{*} B D(\Omega) \times \mathbb{M}_{b}\left(\Gamma_{1}, \mathbb{R}^{n}\right)$ topology over bounded subsets of $B D(\Omega) \times \mathbb{M}_{b}\left(\Gamma_{1}, \mathbb{R}^{n}\right)$, for $\mathbf{f}=\mathbf{0}$ and $\mathbf{g}=\mathbf{0}$. The functional

$$
\mathbb{M}_{b}\left(\Gamma_{1}, \mathbb{R}^{n}\right) \ni \boldsymbol{\mu} \mapsto \int_{\Gamma_{1}} \mathbf{g} \cdot \boldsymbol{\mu} \in \mathbb{R}
$$

is continuous in the weak $\mathbb{M}_{b}\left(\Gamma_{1}, \mathbb{R}^{n}\right)$ topology, because $\mathbf{g} \in C\left(\bar{\Gamma}_{1}, \mathbb{R}^{n}\right)$ (cf. Assumption 5). By [2, Lemma 15] the functional

$$
B D(\Omega) \ni \mathbf{u} \mapsto \int_{\Omega} \mathbf{f} \cdot \mathbf{u} d x \in \mathbb{R}
$$

is continuous in the weak* $B D(\Omega)$ topology on bounded subsets of $B D(\Omega)$. If $d s\left(\Gamma_{0}\right) \neq 0$, then by Assumption 7, every minimizing sequence for $\left(S P_{0, j}^{* *}\right)_{A L}$ is bounded.

If $d s\left(\Gamma_{0}\right)=0$, then the assertion of Corollary 10 holds for (3.8) from [2] and $\left(S P_{\lambda, j}^{* *}\right)$, where $j$ is replaced by $j_{\infty}$. Then $\inf \left(S P_{0, j}^{* *}\right)_{A L}$ is equal to the infimum in the limit analysis problem associated to $\left(P_{\lambda, j_{\infty}}\right)$, defined by

$$
\begin{aligned}
& \left(P_{0, j}\right)_{A L} \text { Find inf }\left\{\int_{\Omega} j_{\infty}(x, \boldsymbol{\varepsilon}(\mathbf{u})) d x \mid \mathbf{u} \in L D(\Omega),\right. \\
& \left.\boldsymbol{\gamma}_{B}(\mathbf{u})=\mathbf{0} \text { on } \Gamma_{0} \text { and } L(\mathbf{u})=1\right\}
\end{aligned}
$$

(cf. (3.2)). There exists a bounded minimizing net $\left\{\widetilde{\mathbf{u}}_{k}\right\}_{k \in \mathbf{K}}$ for $\left(P_{0, j}\right)_{A L}$, since $G_{j_{\infty}}(\varepsilon(\cdot))$ does not depend on $u_{s} \in \operatorname{ker} \varepsilon=\mathcal{R}_{0}$ and $L(\widehat{\mathbf{u}})=0$ for every $\widehat{\mathbf{u}} \in \mathcal{R}_{0}$. Therefore $\left\{\left(\widetilde{\mathbf{u}}_{k}, \gamma_{B}\left(\widetilde{\mathbf{u}}_{k}\right)\right\}_{k \in \mathbf{K}}\right.$ is a bounded minimizing net for $\left(S P_{0, j}^{* *}\right)_{A L}$.

Then by continuity (in the weak $B D(\Omega) \times \mathbb{M}_{b}\left(\Gamma_{1}, \mathbb{R}^{n}\right)$ topology) of the functionals (3.9) and (3.10) over bounded sets in $B D(\Omega) \times \mathbb{M}_{b}\left(\Gamma_{1}, \mathbb{R}^{n}\right)$, we obtain the existence of the minimum for $\left(S P_{0, j}^{* *}\right)_{A L}$.

4. Appendix. Below we introduce a family of perturbations studied in convex optimization (see [9, Chapter 3]). Next, we apply these results to Hencky plasticity.

Let $V$ and $V^{*}$ (resp. $Y$ and $Y^{*}$ ) be two topological vector spaces in duality via the bilinear pairing $\langle\cdot, \cdot\rangle_{V}\left(\langle\cdot, \cdot\rangle_{Y}\right.$, respectively). We shall assume the existence of a continuous linear operator $\Lambda$ from $V$ into $Y$, with transpose $\Lambda^{*}$. Taking a function $V \ni \mathbf{u} \mapsto F(\mathbf{u})+G(\Lambda \mathbf{u}) \in \mathbb{R} \cup\{\infty\}$, we are concerned with the minimization problem

$$
(P) \quad \inf \{F(\mathbf{u})+G(\Lambda \mathbf{u}) \mid \mathbf{u} \in V\} .
$$

We shall also consider a function $\Phi: V \times Y \rightarrow \mathbb{R} \cup\{\infty\}$ such that $\Phi(\mathbf{u}, \mathbf{0})=F(\mathbf{u})+G(\Lambda \mathbf{u})$, and for every $\mathbf{z} \in Y$ we shall consider the mini- 
mization problem

$$
\left(P_{\mathbf{z}}\right) \quad \inf \{\Phi(\mathbf{u}, \mathbf{z}) \mid \mathbf{u} \in V\},
$$

where $\Phi(\mathbf{u}, \mathbf{z}) \equiv F(\mathbf{u})+G(\Lambda(\mathbf{u})+\mathbf{z})$ for every $\mathbf{u} \in V$ and $\mathbf{z} \in Y$. The problems $\left(P_{\mathbf{z}}\right)$ will be said to be perturbed problems of $(P)$. Let $\Phi^{*}$ be the conjugate function of $\Phi$ in the duality between $V \times Y$ and $V^{*} \times Y^{*}$, given by

$$
\begin{gathered}
\Phi^{*}\left(\mathbf{u}^{*}, \mathbf{z}^{*}\right)=\sup \left\{\left\langle\mathbf{u}^{*}, \mathbf{u}\right\rangle_{V}-F(\mathbf{u})-\left\langle\mathbf{z}^{*}, \Lambda \mathbf{u}\right\rangle_{Y}+\left\langle\mathbf{z}^{*}, \Lambda(\mathbf{u})+\mathbf{z}\right\rangle_{Y}\right. \\
-G(\Lambda(\mathbf{u})+\mathbf{z}) \mid \mathbf{u} \in V, \mathbf{z} \in Y\}=F^{*}\left(\mathbf{u}^{*}-\Lambda^{*} \mathbf{z}^{*}\right)+G^{*}\left(\mathbf{z}^{*}\right) .
\end{gathered}
$$

The problem

$$
\left(P^{*}\right) \quad \sup \left\{-\Phi^{*}\left(\mathbf{0}, \mathbf{z}^{*}\right) \mid \mathbf{z}^{*} \in Y^{*}\right\}
$$

is termed the dual problem of $(P)$ with respect to $\Phi$.

It is natural to associate the perturbed problems $\left(\mathbf{u}^{*} \in V^{*}\right)$

$$
\left(P_{\mathbf{u}^{*}}^{*}\right) \sup \left\{-\Phi^{*}\left(\mathbf{u}^{*}, \mathbf{z}^{*}\right) \mid \mathbf{z}^{*} \in Y^{*}\right\}
$$

with the dual problem $\left(P^{*}\right)$, and to determine the dual problem of $\left(P^{*}\right)$ with respect to these perturbations; we easily arrive at the following problem which will be termed the bidual problem of $(P)$ :

$$
\left(P^{* *}\right) \quad \inf \left\{\Phi^{* *}(\mathbf{u}, \mathbf{0}) \mid \mathbf{u} \in V\right\},
$$

where $\Phi^{* *}(\mathbf{u}, \mathbf{z})=F^{* *}(\mathbf{u})+G^{* *}(\Lambda(\mathbf{u})+\mathbf{z})$ for every $\mathbf{u} \in V, \mathbf{z} \in Y$ and

$$
\begin{aligned}
F^{* *}(\mathbf{u}) & =\sup \left\{\left\langle\mathbf{u}^{*}, \mathbf{u}\right\rangle_{V}-F^{*}\left(\mathbf{u}^{*}\right) \mid \mathbf{u}^{*} \in V^{*}\right\}, \\
G^{* *}(\Lambda(\mathbf{u})+\mathbf{z}) & =\sup \left\{\left\langle\mathbf{z}^{*}, \Lambda(\mathbf{u})+\mathbf{z}\right\rangle_{Y}-G^{*}\left(\mathbf{z}^{*}\right) \mid \mathbf{z}^{*} \in Y^{*}\right\} .
\end{aligned}
$$

Below we study Hencky plasticity with the Mises (or Tresca) yield condition (see [10]). We assume that $\Omega \subset \mathbb{R}^{n}$ and $n \geq 2$.

Assumption 9. Let $\mathcal{K}^{D}: \bar{\Omega} \rightarrow 2^{\left(\mathbf{E}_{s}^{n}\right)^{D}}$ be a multifunction such that $\mathcal{K}^{D}(x) \subset\left(\mathbf{E}_{s}^{n}\right)^{D}$ and

$$
\mathcal{K}(x)=\mathcal{K}^{D}(x) \oplus\left\{\mathbf{w}^{*} \in \mathbf{E}_{s}^{n} \mid \exists t \in \mathbb{R}, w_{i j}^{*}=t \delta_{i j}\right\}
$$

for every $x \in \bar{\Omega}$, where $\left(\mathbf{E}_{s}^{n}\right)^{D}$ denotes the set of symmetric real $n \times n$ matrices (whose trace is 0 ) and $w_{i j}^{*}$ are the components of $\mathbf{w}^{*}$. Here $\delta_{i j}=1$ if $i=j$ and $\delta_{i j}=0$ otherwise. There exist $r_{1}, r_{2}>0$ such that

$$
B_{\left(\mathbf{E}_{s}^{n}\right)^{D}}\left(0, r_{1}\right) \subset \mathcal{K}^{D}(x) \subset B_{\left(\mathbf{E}_{s}^{n}\right)}\left(0, r_{2}\right) .
$$

Assumption 10. The normal integrand $j^{*}$ satisfies

$$
j^{*}\left(x, \mathbf{w}^{*}\right)=j_{D}^{*}\left(x,\left(\mathbf{w}^{*}\right)^{D}\right)+j_{\operatorname{tr}}^{*}\left(x, \frac{1}{n} \operatorname{tr} \mathbf{w}^{*}\right)
$$


for every $\mathbf{w}^{*} \in \mathbf{E}_{s}^{n}$ and $d x$-a.e. $x \in \Omega$, where $j_{D}^{*}$ and $j_{t r}^{*}$ are nonnegative functions, and the deviator $\left(\mathbf{w}^{*}\right)^{D}$ of $\mathbf{w}^{*}$ is given by

$$
w_{i j}^{* D}=w_{i j}^{*}-\frac{1}{n} \delta_{i j}\left(\operatorname{tr} \mathbf{w}^{*}\right) .
$$

There exist $\widehat{k}>0, a \geq a_{1}>0$ and $b \geq 0$ such that

$$
\begin{gathered}
a t^{2}+b \geq j_{\mathrm{tr}}^{*}(x, t) \geq a_{1} t^{2} \quad \forall t \in \mathbb{R}, \text { for } d x \text {-a.e. } x \in \Omega \\
j_{D}^{*}\left(x,\left(\mathbf{w}^{*}\right)^{D}\right) \leq \widehat{k} \quad \forall\left(\mathbf{w}^{*}\right)^{D} \in \mathcal{K}^{D}(x), \text { for } d x \text {-a.e. } x \in \Omega .
\end{gathered}
$$

The vector spaces

$$
\begin{aligned}
U(\Omega) & \equiv\left\{\mathbf{u} \in B D(\Omega) \mid \operatorname{div} \mathbf{u} \in L^{2}(\Omega)\right\}, \\
U_{p}(\Omega) & \equiv\left\{\mathbf{u} \in B D(\Omega) \mid \operatorname{div} \mathbf{u} \in L^{n /(n-1)}(\Omega)\right\}
\end{aligned}
$$

are Banach spaces with the natural norms

$$
\begin{aligned}
\|\mathbf{u}\|_{U(\Omega)} & =\|\mathbf{u}\|_{B D(\Omega)}+\|\operatorname{div} \mathbf{u}\|_{L^{2}(\Omega)}, \\
\|\mathbf{u}\|_{U_{p}(\Omega)} & =\|\mathbf{u}\|_{B D(\Omega)}+\|\operatorname{div} \mathbf{u}\|_{L^{n /(n-1)}(\Omega)}
\end{aligned}
$$

(see [13]). We obtain

$$
U(\Omega) \subset U_{p}(\Omega) \subset B D(\Omega),
$$

since $\Omega \subset \mathbb{R}^{n}$ is a bounded set and $n \geq 2$. Moreover, we consider the space (4.20) $\Sigma(\Omega) \equiv\left\{\boldsymbol{\sigma} \in L^{2}\left(\Omega, \mathbf{E}_{s}^{n}\right) \mid \boldsymbol{\sigma}^{D} \in L^{\infty}\left(\Omega, \mathbf{E}_{s}^{n}\right), \operatorname{div} \boldsymbol{\sigma} \in L^{n+1}\left(\Omega, \mathbb{R}^{n}\right)\right\}$ endowed with the natural norm

$$
\|\boldsymbol{\sigma}\|_{\Sigma(\Omega)}=\left\|\boldsymbol{\sigma}^{D}\right\|_{L^{\infty}\left(\Omega, \mathbf{E}_{s}^{n}\right)}+\|\boldsymbol{\sigma}\|_{L^{2}\left(\Omega, \mathbf{E}_{s}^{n}\right)}+\|\operatorname{div} \boldsymbol{\sigma}\|_{L^{n+1}\left(\Omega, \mathbb{R}^{n}\right)}
$$

(cf. [13, Chapter 2, Section 7]). Define

$$
\Sigma_{s}(\Omega) \equiv\left\{\boldsymbol{\sigma} \in \Sigma(\Omega) \mid \boldsymbol{\gamma}_{\Sigma}(\boldsymbol{\sigma}) \in L^{\infty}\left(\operatorname{Fr} \Omega, \mathbb{R}^{n}\right)\right\},
$$

where the trace $\gamma_{\Sigma}: \Sigma(\Omega) \rightarrow C^{1}\left(\operatorname{Fr} \Omega, \mathbb{R}^{n}\right)^{\prime}$ is given by

$$
\gamma_{\Sigma}(\boldsymbol{\sigma})=\boldsymbol{\sigma} \cdot \boldsymbol{\nu} \quad \text { on } \operatorname{Fr} \Omega \text {, for all } \boldsymbol{\sigma} \in C^{1}\left(\bar{\Omega}, \mathbf{E}_{s}^{n}\right) .
$$

Let

$$
V \equiv U_{p}(\Omega), \quad Y^{*} \equiv \Sigma_{s}(\Omega) .
$$

Let $\mathbf{I}$ denote the $n \times n$ unit matrix and set

$$
\begin{array}{r}
Y(\Omega) \equiv \operatorname{span}\left(\varepsilon\left(U_{p}(\Omega)\right), L^{1}\left(\Omega,\left(\mathbf{E}_{s}^{n}\right)^{D}\right) \oplus L^{n /(n-1)}(\Omega, \mathbb{R}) \mathbf{I}\right) \\
=\left\{\boldsymbol{\mu} \in \mathbb{M}_{b}\left(\Omega, \mathbf{E}_{s}^{n}\right) \mid \exists \mathbf{u} \in U_{p}(\Omega)\right. \text { and } \\
\quad \exists \mathbf{w} \in L^{1}\left(\Omega,\left(\mathbf{E}_{s}^{n}\right)^{D}\right) \oplus L^{n /(n-1)}(\Omega, \mathbb{R}) \mathbf{I} \\
\quad \text { such that } \boldsymbol{\mu}=\boldsymbol{\varepsilon}(\mathbf{u})+\mathbf{w} d x\} .
\end{array}
$$

The dual space $V^{*}$ (to $\left.U_{p}(\Omega)\right)$ is

$$
V^{*} \equiv\left\{\left(\mathbf{u}^{*}, \boldsymbol{\psi}^{*}\right) \in L^{n}\left(\Omega, \mathbb{R}^{n}\right) \times L^{\infty}\left(\operatorname{Fr} \Omega, \mathbb{R}^{n}\right)\right\} .
$$


The bilinear form between $U_{p}(\Omega)$ and $V^{*}$ is

$$
\begin{aligned}
U_{p}(\Omega) \times V^{*} \ni\left(\mathbf{u},\left(\mathbf{u}^{*}, \boldsymbol{\psi}^{*}\right)\right) & \mapsto-\int_{\Omega} \mathbf{u}^{*} \cdot \mathbf{u} d x \\
& +\int_{\operatorname{Fr} \Omega} \boldsymbol{\psi}^{*} \cdot \boldsymbol{\gamma}_{B}(\mathbf{u}) d s \equiv\left\langle\mathbf{u} ;\left(\mathbf{u}^{*}, \boldsymbol{\psi}^{*}\right)\right\rangle_{U_{p} \times V^{*}} .
\end{aligned}
$$

The space $U_{p}(\Omega)$ is endowed with the topology $\sigma\left(U_{p}(\Omega), V^{*}\right)$. Similarly $V^{*}$ is endowed with the topology $\sigma\left(V^{*}, U_{p}(\Omega)\right)$. Therefore $\left[U_{p}(\Omega), \sigma\left(U_{p}(\Omega), V^{*}\right)\right]^{*}$ $=V^{*}$ and $\left[V^{*}, \sigma\left(V^{*}, U_{p}(\Omega)\right)\right]^{*}=U_{p}(\Omega)$ (see [8, Theorem V.3.9]).

The bilinear form between $Y$ and $Y^{*}$ is given by

$$
\langle\boldsymbol{\mu} ; \boldsymbol{\sigma}\rangle_{Y \times Y^{*}}=\int_{\Omega} \boldsymbol{\sigma}: \boldsymbol{\mu}=\int_{\Omega} \boldsymbol{\sigma}: \boldsymbol{\varepsilon}(\mathbf{u})+\int_{\Omega} \boldsymbol{\sigma}: \mathbf{w} d x
$$

for $\boldsymbol{\sigma} \in \Sigma_{s}(\Omega)$ and $\boldsymbol{\mu} \in Y$, where $\boldsymbol{\mu}=\boldsymbol{\varepsilon}(\mathbf{u})+\mathbf{w}, \mathbf{u} \in U_{p}(\Omega)$ and $\mathbf{w} \in$ $L^{1}\left(\Omega,\left(\mathbf{E}_{s}^{n}\right)^{D}\right) \oplus L^{n /(n-1)}(\Omega, \mathbb{R}) \mathbf{I}$. Then we have $\left[Y, \sigma\left(Y, Y^{*}\right)\right]^{*}=Y^{*}$ and $\left[Y^{*}, \sigma\left(Y^{*}, Y\right)\right]^{*}=Y$.

The linear operator $\varepsilon:\left[U_{p}, \sigma\left(U_{p}, V^{*}\right)\right] \rightarrow\left[Y, \sigma\left(Y, \Sigma_{s}(\Omega)\right)\right]$ is continuous. The explicit form of $\varepsilon^{*}: \Sigma_{s}(\Omega) \rightarrow V^{*}$ reads

$$
\begin{aligned}
\langle\varepsilon(\mathbf{u}), \boldsymbol{\sigma}\rangle_{Y \times Y^{*}} & =\int_{\Omega} \boldsymbol{\sigma}: \varepsilon(\mathbf{u})=\int_{\Omega} \boldsymbol{\sigma}^{D}: \varepsilon^{D}(\mathbf{u})+\frac{1}{n} \int_{\Omega} \operatorname{tr} \boldsymbol{\sigma} \operatorname{div} \mathbf{u} d x \\
& =-\int_{\Omega}(\operatorname{div} \boldsymbol{\sigma}) \cdot \mathbf{u} d x+\int_{\operatorname{Fr} \Omega} \gamma_{\Sigma}(\boldsymbol{\sigma}) \cdot \gamma_{B}(\mathbf{u}) d s \\
& =\left\langle\mathbf{u} ; \varepsilon^{*}(\boldsymbol{\sigma})\right\rangle_{U_{p} \times V^{*}} .
\end{aligned}
$$

Hence the function $\varepsilon^{*}: \Sigma_{s}(\Omega) \rightarrow V^{*}$ is given by $\varepsilon^{*}(\boldsymbol{\sigma})=\left(\operatorname{div} \boldsymbol{\sigma}, \gamma_{\Sigma}(\boldsymbol{\sigma})\right)$.

The original problem $\left(P_{\lambda, j}\right)$ is defined by $(3.1)-(3.4)$.

Let the functional of relaxed elastic-plastic energy $\left\lceil R P_{\lambda, j}\right\rceil: B D(\Omega) \rightarrow$ $\mathbb{R} \cup\{\infty\}$ be defined by

$$
\left\lceil R P_{\lambda, j}\right\rceil(\mathbf{u})=\lambda F_{R}(\mathbf{u})+G_{j}(\varepsilon(\mathbf{u})),
$$

where $\lambda F_{R}: B D(\Omega) \rightarrow \mathbb{R} \cup\{\infty\}$ is defined by

$$
\lambda F_{R}(\mathbf{u}) \equiv-\lambda L(\mathbf{u})+\int_{\Gamma_{0}} j_{\infty}\left(x,-\gamma_{B}(\mathbf{u}) \otimes_{s} \boldsymbol{\nu}\right) d s
$$

if $\mathbf{u} \in L D(\Omega)$, and $\lambda F_{R}(\mathbf{u}) \equiv \infty$ otherwise (cf. (3.2)). The elastic-plastic potential $G_{j}$ is given by (3.4). The relaxed problem $\left(R P_{\lambda, j}\right)$ is

$$
\left(R P_{\lambda, j}\right) \text { find } \inf \left\{\left\lceil R P_{\lambda, j}\right\rceil(\mathbf{u}) \mid \mathbf{u} \in U_{p}(\Omega)\right\} .
$$

We can show that the dual relaxed problem $\left(R P_{\lambda, j}^{*}\right)$ is

$$
\begin{aligned}
\left(R P_{\lambda, j}^{*}\right) \quad \sup \left\{-\int_{\Omega} j^{*}(x, \boldsymbol{\sigma}) d x \mid \boldsymbol{\sigma} \in \Sigma_{s}(\Omega),\right. \\
\left.\quad \operatorname{div} \boldsymbol{\sigma}=-\lambda \mathbf{f} \text { in } \Omega \text { and } \gamma_{\Sigma}(\boldsymbol{\sigma})=\lambda \mathbf{g} \text { on } \Gamma_{1}\right\} .
\end{aligned}
$$


The bidual relaxed problem $\left(R P_{\lambda, j}^{* *}\right)$ is

$$
\left(R P_{\lambda, j}^{* *}\right) \text { find } \inf \left\{\left(\lambda F_{R}\right)^{* *}(\mathbf{u})+G_{j}^{* *}(\varepsilon(\mathbf{u})) \mid \mathbf{u} \in U_{p}(\Omega)\right\},
$$

where $\left(\lambda F_{R}\right)^{* *}(\mathbf{u})=\lambda F_{R}(\mathbf{u})$ for every $\mathbf{u} \in U_{p}(\Omega)$ and

$$
G_{j}^{* *}(\boldsymbol{\varepsilon}(\mathbf{u}))=\int_{\Omega} j\left(x, \boldsymbol{\varepsilon}(\mathbf{u})_{a}\right) d x+\int_{\Omega} j_{\infty}\left(x, \frac{d \boldsymbol{\varepsilon}(\mathbf{u})_{s}}{d\left|\boldsymbol{\varepsilon}(\mathbf{u})_{s}\right|}\right) d\left|\boldsymbol{\varepsilon}(\mathbf{u})_{s}\right|
$$

for every $\mathbf{u} \in U_{p}(\Omega)$.

Similarly to [14], we prove that the supremum in the problem $\left(R P_{\lambda, j}^{*}\right)$ exists in the space $\Sigma(\Omega)$. Moreover, if $d s\left(\Gamma_{0}\right)=0$, the solution of $\left(R P_{\lambda, j}^{*}\right)$ belongs to the space $\Sigma_{s}(\Omega)$, since $\mathbf{g} \in C^{1}\left(\Gamma_{1}, \mathbb{R}^{n}\right)$.

In [13] and [1] an existence theorem for $\left(R P_{\lambda, j}^{* *}\right)$ is proved in the space $U(\Omega)$ (cf. (4.11), (4.13), (4.14) and (4.19)).

By $\left[9\right.$, Chapter 3], the dual problem to $\left(R P_{\lambda, j}^{* *}\right)$ is equal to $\left(R P_{\lambda, j}^{*}\right)$.

If $\widehat{\boldsymbol{\sigma}}$ is a solution of $\left(R P_{\lambda, j}^{*}\right), \widehat{\mathbf{u}}$ is a solution of $\left(R P_{\lambda, j}^{* *}\right), \inf \left(R P_{\lambda, j}^{* *}\right)=$ $\sup \left(R P_{\lambda, j}^{*}\right)$ and this value is finite, then the couple $(\widehat{\boldsymbol{\sigma}}, \widehat{\mathbf{u}})$ satisfies the extremality relation (see [9, Chapter 3, Proposition 2.4]).

\section{References}

[1] J. L. Bojarski, The relaxation of Signorini problems in Hencky plasticity, I: threedimensional solid, Nonlinear Anal. 29 (1997), 1091-1116.

[2] -, General method of regularization. I: functionals defined on BD space, Appl. Math. (Warsaw) 31 (2004), 175-199.

[3] G. Bouchitté, I. Fonseca and L. Mascarenhas, Relaxation of variational problems under trace constraints, Nonlinear Anal. 49 (2002), 221-246.

[4] G. Bouchitté et P. M. Suquet, Charges limites, plasticité et homogénéisation : le cas d'un bord chargé, C. R. Acad. Sci. Paris Sér. I Math. 305 (1987), 441-444.

[5] G. Bouchitté and M. Valadier, Integral representation of convex functionals on a space of measures, J. Funct. Anal. 80 (1988), 398-420.

[6] E. Christiansen, On the collapse solution in limit analysis, Arch. Rat. Mech. Anal. 91 (1986), 119-135.

[7] -, Limit analysis in plasticity as a mathematical programming problem, Calcolo 17 (1980), 41-65.

[8] N. Dunford and J. T. Schwartz, Linear Operators, Part I, Interscience, New York, 1958.

[9] I. Ekeland and R. Temam, Convex Analysis and Variational Problems, NorthHolland, Amsterdam, 1976.

[10] Y. C. Fung, Foundations of Solid Mechanics, Prentice-Hall, Englewood Cliffs, NJ, 1965.

[11] M. Giaquinta, G. Modica and J. Souček, Cartesian Currents in the Calculus of Variations. Vol. II: Variational Integrals, Ergeb. Math. Grenzgeb. 38, Springer, Berlin, 1998.

[12] P. M. Suquet, Discontinuities and plasticity, in: Non-smooth Mechanics and Applications, J. J. Moreau and P. D. Panagiotopoulos (eds.), Springer, Wien, 1988, $278-340$. 
[13] R. Temam, Mathematical Problems in Plasticity, Gauthier-Villars, Paris, 1985.

[14] R. Temam and G. Strang, Duality and relaxation in the variational problems of plasticity, J. Mécanique 19 (1980), 493-527.

Institute of Fundamental Technological Research

Polish Academy of Sciences

Świętokrzyska 21

00-049 Warszawa, Poland

E-mail: jbojar@ippt.gov.pl

Received on 28.1.2003;

revised version on 10.2.2004 